Draft Version November 12, 2018

Typeset using IATEX default style in AASTeX61

\title{
MODELING THE VARIABILITY OF ACTIVE GALACTIC NUCLEI BY INFINITE MIXTURE OF ORNSTEIN-UHLENBECK(OU) PROCESSES
}

\author{
Tadafumi Takata, ${ }^{1,2}$ Yusuke Mukuta, ${ }^{3}$ And Yoshikiko Mizumoto ${ }^{4}$ \\ ${ }^{1}$ Astronomy Data Center, National Astronomical Observatory of Japan, National Institutes of Natural Science(NINS) Osawa 2-21-1 \\ Tokyo, 181-8588, JAPAN \\ 2 The Graduate University for Advanced Study (SOKENDAI) \\ ${ }^{3}$ Graduate School of Information Science and Technology, The University of Tokyo \\ ${ }^{4}$ National Astronomical Observatory of Japan, National Institutes of Natural Science(NINS)
}

\begin{abstract}
We develop an infinite mixture model of Ornstein-Uhlenbeck(OU) processes for describing the optical variability of QSOs based on treating the variability as a stochastic process. This enables us to get the parameters of the power spectral densities(PSDs) on their brightness variations by providing more flexible description of PSDs than the models based on single OU process(damped random walk). We apply this model to 67,507 variable objects extracted from SDSS Stripe82 photometric data and succeed in showing very high precision in identifying QSOs ( $~ 99 \%$ levels in completeness and purity) among variable objects based only on their variability, by investigating on 9,855 spectroscopically confirmed objects(7,714 QSOs and 2,141 stars) in the data of SDSS Data Release 12(DR12), with sufficient and accurate multiple measurements of their brightness. By comparing our results with the values based on other models that are used in previous research, it is revealed that our model can be used as the most effective method for selecting QSOs from variable object catalog, especially regarding completeness and purity. The main reason of improved identification rates are the ability of our model to separate clearly QSOs and stars, especially on the small fraction of QSOs with variabilities which can be described better than simple damped random walk model.
\end{abstract}

Keywords: Active Galactic Nuclei(AGN) — Quasi Stellar Objects(QSOs) — optical variability — stochastic process — Ornstein-Uhlenbeck(OU) Process — classification 


\section{INTRODUCTION}

One of the remarkable properties of active galactic nuclei (AGNs), frequently represented by Quasi Stellar Objects(QSOs), is their variability seen in a wide range of the electromagnetic wave region. Just after the discovery of AGNs(Schmidt 1963; Matthews \& Sandage 1963), their variability became well known(Greenstein 1963; Smith \& Hoffleit 1963) and studied by many authors writing about theory and observational data analysis. Since Sesar et al. (2007) demonstrated that at least $90 \%$ of QSOs show the signature of variability with rms more than 0.03 mag, it is believed that most QSO/AGN populations have a feature of variability. As AGNs are among the most luminous celestial objects in the universe, their variability with an order of $10 \%$ of their total light in various time scales (less than one hour to many years)(Gaskell \& Klimek 2003; Uttley \& Casella 2014), are one of the largest fluctuations in energy, and discovering their originating physical processes is an attractive quest. Because the optical continuum radiation is believed to be predominantly coming from the accretion disk, it is straightforward to consider that some processes intrinsic to the disk are the origin of the brightness fluctuations. There is such a lot of theoretical work discussing the origin of the variability, in the short and long term, based on the change of global accretion rate(for example, Pereyra et al. (2006)), disk inhomogeneities propagating inward(for example, Dexter \& Agol (2011)), and these fluctuations may arise from thermal or magneto-rotational instabilities in a turbulent accretion flow(Hirose et al. 2009; Jiang et al. 2013), though it is still unknown what physical processes control their variability.

On the data analysis side, there is still a lot of research describing their behaviors by using structure functions(SFs) and power spectral densities(PSDs). In recent years, there has been much progress in the modeling of their variability (light curves) by means of massive data with long duration and/or dense cadences. There are many extensive studies on QSO/AGN variability, as a powerful tool for QSO selection using legacy and/or newly acquired data, and also as a probe for physical models of AGNs (Kelly et al. 2009, 2011, 2013; Kozlowski et al. 2010a, 2011, 2012; Kozlowski 2016b; MacLeod et al. 2010, 2011, 2012; Schmidt et al. 2010, 2012; Palanque-Delabrouille et al. 2012; Butler \& Bloom 2011; Kim et al. 2011; Ruan et al. 2012; Zuo et al. 2012; Andrae et al. 2013; Zu et al. 2013; Morganson et al. 2014; Graham et al. 2014; De Cicco et al. 2015; Falocco et al. 2015; Cartier et al. 2015; Caplar et al. 2017).

A model with particularly successful results is the damped random walk (DRW) model. This model was first introduced by Press et al. (1992) and Rybicki \& Press (1992), and the fast computational implementation was described by Rybicki \& Press (1995), for inferring the time lag of variability among multiply imaged gravitationally lensed QSOs. With the detailed analysis by Kelly et al. (2009), Kozlowski et al. (2010a) and MacLeod et al. (2010) on various time series data, it is established that a DRW model can statistically explain the observed light curves of QSOs at the enough fidelity level(0.01 to $0.02 \mathrm{mag})$. Although the DRW model is relatively simple in description, it successfully describes the most of QSOs' light curves, leading to very high rates in identifying QSOs from the variable sources especially in optical wavelength(for example, Kozlowski et al. (2010a); MacLeod et al. (2010, 2011); Choi et al. (2014)), although it is revealed to be applicable also to mid-infrared data(Kozlowski et al. 2010b; Kozlowski 2016b). It should also be noted that the confidence level and completeness of QSO selection will be increased by the combination of colors and variability(Peters et al. 2015), and/or with support by image subtraction technique(Choi et al. 2014). The model calculation is relatively fast; it needs computational times only scaling to the number of data points $(O(N)$ in case $\mathrm{N}$ is the number of data points), by the implementation of SF for DRW model by using auto-correlation function(MacLeod et al. 2010; Butler \& Bloom 2011), although the other implementation using Markov Chain Monte Carlo (MCMC) for reproducing the continuous light curves and exponential covariance matrix takes longer in $O\left(N^{2}\right)$ (Zu et al. 2011, 2013). On the other hand, there are claims that DRW is too simple to describe the 'real' variability of QSOs/AGNs, because the possibility of the degeneracies cannot be eliminated(Kozlowski 2016c). Kasliwal et al. (2015a) investigated the optical light curves of 20 QSOs observed with Kepler satellite, and found that fewer than half of them can be explained by the DRW model.

Thus there are some alternatives for describing the variability in Fourier space with PSDs, by the model with mixture of Ornstein-Uhlenbech(OU) processes(Kelly et al. 2011), Slepian wavelet variance(Graham et al. 2014), continuous auto regression and moving average(CARMA) model(Kelly et al. 2014; Simm et al. 2016; Kasliwal et al. 2017), broken power law model(Zhu \& Xue 2016) and so on.

The OU mixture model by Kelly et al. (2011) is one of the powerful candidates of QSO/AGN variability modelings because it is very natural to try to express the fluctuation phenomena by the combination of the simple random processes. The method of the OU mixture is based on the long-term accumulation of experience learned in many studies of QSO/AGN and also of galactic objects' variability. As this model treats the variability in Fourier space, we can learn their PSDs, which provide us with the information on how they vary. This should be very useful for 
investigating the physical processes which control the light variations. It is also revealed to be possible to apply to some wavelength data such as X-ray and optical data(Kelly et al. 2011). However, the model has some weak points; the first one is their time-consuming calculation, and the second one is the arbitrariness in the selection of the number of OU processes to be mixed. As suggested in Kelly et al. (2011), the number of mixed OU processes (expressed as $M$ hereafter) should be larger than 30 to describe the sufficiently observed AGN's light curves, and the calculation is too massive for large sample data, because the calculation time is on the order of $O\left(M N^{2}\right)$, which is too massive for those for DRW model calculations.

We've tried to overcome these weak points by extending the mixture numbers of OU processes to infinite, and succeeded in finishing the calculation in much less time than the original model and without the arbitrariness in OU mixture. By applying our infinite OU mixture model to 67,507 variable sources extracted from the photometric data of Stripe 82 in Sloan Digital Sky Survey(SDSS), we confirm the ability of our model in selection of QSOs from various variable objects and compare our results with those by other models previously suggested as successful. By our model we can show the variation of the PSDs of numerous $(\sim 8,000)$ spectroscopically confirmed QSOs with sufficient multiple photometric measurements during $\sim 10$ years, and provide some implication for the power of PSDs in classifying QSOs/AGNs into subclasses and find some rare type QSOs/AGNs.

It should be emphasized here that we know it is very difficult to describe the details on intrinsic variability and the underlying physical processes of individual QSOs in this study, as the data of SDSS Stripe82 suffer from irregular and sparse time sampling, and our model uses approximate PSD model. Therefore, our main purpose in this paper is to know where is the limit in distinguishing QSOs from stars only using their variability, with superpositions of OU processes, which is more flexible to describe variability of QSOs/AGNs than those based on single OU process.

In section 2, we will describe the methodology by which we construct our model, and describe the data used for our test in section 3. In section 4, we will show the results by our analysis of Stripe 82 data. We show the methods on classification and identification rates based on variability in section 5 , discuss the features of our model in section 6 , and provide the conclusions in section 7 .

\section{METHODOLOGIES}

In this section, we describe existing models and our proposed models as their limit. We first describe the estimation method common to each model in 2.1. We describe OU process in 2.2, and the model with finite mixture of OU process proposed by Kelly et al. (2011) in 2.3. Afterward we describe our model with infinite mixture of OU processes in 2.4, and practical implementation and model calculation in 2.5 .

\subsection{Estimation Method}

For estimating the best model parameters, by which the physical process is described, we usually perform fitting by using maximum-likelihood or Bayesian techniques. There is an advantage in estimating the parameters directly from the light curve by using all of the information in the data. It is especially useful to estimate the parameters of power spectrum model as they are not heavily biased by measurement errors, irregular sampling, or other windowing effects caused by the finite time span of the light curve, such as red noise leak(Kelly et al. 2011). Though the likelihood function may be used to calculate a maximum likelihood estimate, we employ a Bayesian approach which calculates the posterior probability distribution of the model parameters, in order to reliably estimate the uncertainties of the model parameters. The probability distribution of the parameters for the given light curve by the observation is

$$
p\left(\theta \mid x_{t_{1}}, \ldots, x_{t_{N}}\right) \propto p\left(x_{t_{1}}, \ldots, x_{t_{N}} \mid \theta\right) p(\theta),
$$

where $p(\theta)$ and $p\left(\theta \mid x_{t_{1}}, \ldots, x_{t_{N}}\right)$ are the prior and the posterior distributions.

The likelihood function $p\left(x_{t_{1}}, \ldots, x_{t_{N}} \mid \theta\right)$ can be written as the following form

$$
p\left(x_{t_{1}}, \ldots, x_{t_{N}} \mid \theta\right)=\frac{1}{\sqrt{(2 \pi)^{N}|C|}} \times \exp \left(-\frac{\mathbf{x}^{T} C^{-1} \mathbf{x}}{2}\right)
$$

, where $C$ is covariance and the vector $\mathbf{x}=\left(x_{t_{1}}, x_{t_{2}}, \ldots, x_{t_{N-1}}, x_{t_{N}}\right)$. Here we denote model parameter as $\theta$ and observed data as $\left\{x_{t_{n}}\right\}_{n=1}^{N}$, where $x_{t_{n}}$ is a brightness(apparent magnitude) at $t_{n}$ and $N$ is the number of observations(measurements). $N$ is assumed to be about $10 \sim 10^{2}$ in the data-set. Given prior distribution $p(\theta)$ and generation model $p\left(x_{t_{1}}, \ldots, x_{t_{N}} \mid \theta\right)$, we use maximum a posteriori(MAP) estimation as described below

$$
\theta_{\mathrm{MAP}}=\operatorname{argmax}_{\theta} p\left(\theta \mid x_{t_{1}}, \ldots, x_{t_{N}}\right)
$$




$$
=\operatorname{argmax}_{\theta} p\left(x_{t_{1}}, \ldots, x_{t_{N}} \mid \theta\right) p(\theta)
$$

to get the model parameter, where $\theta_{\mathrm{MAP}}$ means the parameter set giving the maximum of likelihood for the model.

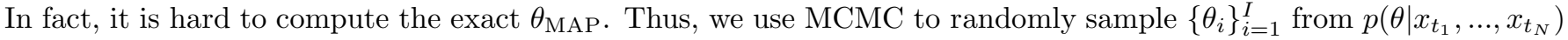
and use the median of $\left\{\theta_{i}\right\}_{i=1}^{I}$ instead, where $I$ is a number of samples. In this work, we use Affine Invariant MCMC Ensemble Sampler(Foreman-Mackey et al. 2012) and set $I=20,000$ for getting statistically stable results by numerous sampling. We tested the cases of $I=5,000,10,000$ and 20,000 and confirmed that there is little difference among the inferred parameter values in the most cases, as mentioned by $\mathrm{Zu}$ et al. (2011). However, as our model will use four parameters, which is twice of those by $\mathrm{Zu}$ et al. (2011), and the model is more complex, we decided to use $I=20,000$ for our calculation.

It requires that we can compute $p\left(x_{t_{1}}, \ldots, x_{t_{N}} \mid \theta\right)$ with small complexity. All models we handle in this paper are Gaussian process. This means that the joint distribution of $\left\{x_{t_{n}}\right\}_{n=1}^{N}$ is a $N$-dimensional Gaussian distribution with $i, j$-element of covariance $C_{i j}=\operatorname{cov}\left(\left|t_{i}-t_{j}\right|, \theta\right)$, where cov is a covariance function. When $C$ is computed, it requires $O\left(N^{3}\right)$ complexity to compute $p\left(x_{t_{1}}, \ldots, x_{t_{N}} \mid \theta\right)$. This does not take much computation because $N$ is small. Thus, our motivation is to propose a covariance function with high representation ability and requires small computational complexity.

\subsection{Damped Random Walk(DRW) Model(OU process)}

The most basic model is OU process. This is a continuous auto-regression model. The model parameter $\theta$ consists of time constant $\tau$ and noise magnitude $\zeta$. OU process is a solution of the following stochastic differential equation:

$$
d X_{t}=-\frac{1}{\tau} X_{t} d t+\zeta d W_{t}
$$

where $W_{t}$ is a white noise.

The covariance function of this model is

$$
\mathcal{R}_{O U}=\frac{\tau \zeta^{2}}{2} e^{\frac{-|\delta t|}{\tau}}
$$

The most important advantage of the OU model is that we need only two parameters for the model $(\zeta$ and $\tau)$. Also, it does not require much computation, as the covariance function is simple. Additionally there is a faster implementation for the model computation by using structure function which is derived from the auto-correlation function of DRW model(Hughes et al. 1992; MacLeod et al. 2010).

The power spectral density can be derived by Fourier transformation of Equation 6,

$$
\begin{aligned}
\mathcal{P}_{O U}(\omega) & =\frac{1}{2 \pi} \int_{-\infty}^{\infty} e^{-i \omega t} \mathcal{R}_{O U} d t \\
& =\frac{\zeta^{2}}{2 \pi} \frac{1}{\omega_{0}^{2}+\omega^{2}},
\end{aligned}
$$

where $\omega_{0}$ is the reciprocal of damping time scale $\tau$, and it is good approximation of majority of QSOs with brightness variation(Kelly et al. 2009; Kozlowski et al. 2010a; MacLeod et al. 2010).

However, since the model is so simple(Kasliwal et al. 2015a; Kozlowski 2016c), the scope of the method is limited. The actual/real process is considered to consist of stochastic process with various scales. Thus, it is natural to consider the mixture model for describing the actual and relatively complex processes.

\subsection{Mixed OU Model}

Kelly et al. (2011) proposed a model for superpositions of OU processes by changing the scale and magnitude of the process, especially for the X-ray light curves. When the number of OU processes is $M$, the model parameters are the lowest and the highest frequencies $\omega_{L}, \omega_{H}$, noise magnitude $\zeta$ and $\alpha$ that determines the magnitudes of each process(weight of mixture). It is noted that the model parameters and method of mixing the OU process is designed to be able to produce the double-bending PSDs, which can be seen in X-ray variability around black holes and also in recent results of QSO variability studies based on the data with more dense cadence such as Kepler, and ground base telescope data (Mushotzky et al. 2011; Carini \& Ryle 2012; Wehrle et al. 2013; Edelson et al. 2014; Graham et al. 2014; Revalski et al. 2014; Kasliwal et al. 2015a,b; Chen \& Wang 2015; Shaya et al. 2015; Kasliwal et al. 2017). 
When we denote $\omega_{k}$ as $k$-th value of $M$ with equal ratio division from $\omega_{L}$ to $\omega_{H}$ and the OU process in Equation 5 with $\tau=\frac{1}{\omega_{k}}$ as $X_{k}$, the mixture model is written as

$$
X=\sum_{k=1}^{M} c_{k} X_{k},
$$

where $c_{k}$ are magnitudes of $X_{k}$ that are proportional to $\omega_{k}^{1-\frac{\alpha}{2}}$ and that $\sum_{k=1}^{M} c_{k}^{2}=1$. This model is regarded as the summation of various OU process with magnitudes depending on the frequency.

Then, the covariance function can be expressed in the following way.

$$
\mathcal{R}_{\text {mix } O U}=\sum_{k=1}^{M} \frac{c_{k}^{2} \zeta^{2}}{2 \omega_{k}} e^{-\omega_{k}|\delta t|} .
$$

Since this is a mixture model of OU processes, it can model a wider variety of processes. The sum of exponentials in Equation 10 falls more slowly than single exponential function. This means that the mixed OU process exhibits longer range dependency than a single OU process and is better suited for modeling the light curves with long timescale dependencies. The power spectral density can also be written in relatively simple form,

$$
\mathcal{P}_{\text {mix } O U}=\sum_{k=1}^{M} \frac{c_{k}^{2} \zeta^{2}}{2 \pi} \frac{1}{\omega_{k}^{2}+\omega^{2}},
$$

where $\omega_{k}=1 / \tau_{k}$ and $\tau_{k}$ is the damping time scale of $k$-th OU process. It should be noted that Kelly et al. (2011) assumes that all of the individual OU processes have the same value of $\zeta$, for avoiding the degeneracy coming from $c_{k}^{2} \zeta_{k}^{2}$, in considering $\zeta_{k}$ as noise magnitude for the $k$-th process. Also, it does not require much complexity when $M$ is small compared to $N$. Kelly et al. (2011) succeeded implementing the efficient calculation by obtaining a state-space representation of the light curve with about 3,000 data points, and then using the Kalman recursions(Brockwell \& Davis 2002).

However, it is not easy to determine the appropriate $M$ beforehand. It is unnatural to assume only $M$ processes contribute to the variability. Considering $M$ as a hyperparameter and trying to search appropriate $M$ by changing $M$ is an option, however it is not realistic because we use the data-set with $10 \sim 10^{2}$ measurements, so it requires too huge amount of time when trying many $M$ values. As it requires the time on the order of $O\left(N M^{2}\right)$ to compute the covariance, it is inefficient in the case of large $M$ relative to $N$. If we can compute the covariance of mixture OU process model with the same time spent for single OU process model, it is very good for applying to large data-set. As described in the next coming sections, it is the case for the data-set we are using this time.

\subsection{Our Model}

In the previous section, we denoted that it is unnatural to determine the number of OU processes $M$ beforehand and that computation is not efficient when $M$ is large. Thus, we try to set $M \rightarrow \infty$ in Equation 10 .

The covariance function of the mixture model described in the Equation 10 can also be written as

$$
\int_{\log \omega_{L}}^{\log \omega_{H}} \frac{c(\omega)^{2} \zeta^{2}}{2 \omega} e^{-\omega|\delta t|} d \log \omega
$$

if written in the form of integral using $\log (\omega)$. As $c(\omega)$ is proportional to $\omega^{1-\frac{\alpha}{2}}$, the equation can be written as

$$
\int_{\omega_{L}}^{\omega_{H}} \frac{A^{2} \omega^{2-\alpha} \zeta^{2}}{2 \omega} e^{-\omega|\delta t|} \frac{d \omega}{\omega}=\int_{\omega_{L}}^{\omega_{H}} \frac{A^{2} \zeta^{2}}{2} \omega^{-\alpha} e^{-\omega|\delta t|} d \omega
$$

by setting $A$ as a constant of proportionality. If we define $x=\omega|\delta t|$,

$$
\frac{A^{2} \zeta^{2}}{2} \int_{\omega_{L}|\delta t|}^{\omega_{H}|\delta t|}\left(\frac{x}{|\delta t|}\right)^{-\alpha} e^{-x} \frac{d x}{|\delta t|}=\frac{A^{2} \zeta^{2}|\delta t|^{\alpha-1}}{2} \Gamma\left(1-\alpha, \omega_{L}|\delta t|, \omega_{H}|\delta t|\right) .
$$

and $A$ can be written as

$$
1=\int_{\log \omega_{L}}^{\log \omega_{H}} A^{2} \omega^{2-\alpha} d \log \omega=\int_{\omega_{L}}^{\omega_{H}} A^{2} \omega^{1-\alpha} d \omega=A^{2} \frac{\omega_{H}^{2-\alpha}-\omega_{L}^{2-\alpha}}{2-\alpha} .
$$


because $\Sigma c_{k}^{2}=1$.

Additionally the integrand of Equation 12 is bounded in the range of $\omega_{L}<\omega<\omega_{H},|\delta t| \geq 0$, we can exchange the integral and $|\delta t| \rightarrow 0$. Therefore we can analytically derive the equation by setting $e^{-\omega|\delta t|}=1$ in the integrand as follows,

$$
\mathcal{R}_{\text {InfOU }}= \begin{cases}\frac{(2-\alpha) \zeta^{2}}{2\left(\omega_{H}^{2-\alpha}-\omega_{L}^{2-\alpha}\right)}|\delta t|^{\alpha-1} \Gamma\left(1-\alpha, \omega_{L}|\delta t|, \omega_{H}|\delta t|\right) & (|\delta t|>0) \\ \frac{\zeta^{2}(2-\alpha)\left(\omega_{H}^{1-\alpha}-\omega_{L}^{1-\alpha}\right)}{2(1-\alpha)\left(\omega_{H}^{2-\alpha}-\omega_{L}^{2-\alpha}\right)} & (|\delta t|=0)\end{cases}
$$

as a covariance function, where $\Gamma\left(1-\alpha, \omega_{L}|\delta t|, \omega_{H}|\delta t|\right)$ is an incomplete gamma function.

We can also derive the power spectral density by using the hyper-geometric function. The power spectrum of the single OU process with a frequency $\omega$ can be written as

$$
\frac{\zeta^{2}}{2 \pi} \frac{1}{\omega^{2}+x^{2}}
$$

Then the power spectrum of our model can be derived by integrating it with $\omega$.

$$
\frac{\zeta^{2}}{2 \pi} \int_{\log \omega_{L}}^{\log \omega_{H}} \frac{c(\omega)^{2}}{\omega^{2}+x^{2}} d \log \omega=\frac{\zeta^{2} A^{2}}{2 \pi} \int_{\omega_{L}}^{\omega_{H}} \frac{\omega^{1-\alpha}}{\omega^{2}+x^{2}} d \omega .
$$

If we define $\omega=x \tan \theta$, the integration can be written as

$$
\frac{\zeta^{2} A^{2}}{2 \pi} \int_{\arctan \left(\frac{\omega_{L}}{x}\right)}^{\arctan \left(\frac{\omega_{H}}{x}\right)} \frac{x^{1-\alpha} \tan ^{1-\alpha} \theta}{x^{2}\left(1+\tan ^{2} \theta\right)} \frac{x d \theta}{\cos ^{2} \theta}=\frac{\zeta^{2} A^{2} x^{-\alpha}}{2 \pi} \int_{\arctan \left(\frac{\omega_{L}}{x}\right)}^{\arctan \left(\frac{\omega_{H}}{x}\right)} \tan ^{1-\alpha} \theta d \theta,
$$

As the integrand of equation 19 can be written as below,

$$
\int \tan ^{1-\alpha}(x) d x=\frac{1}{2-\alpha} \operatorname{Hyper}_{2 \mathrm{~F} 1}\left(1,1-\frac{\alpha}{2}, 2-\frac{\alpha}{2},-\tan ^{2}(x)\right) \tan ^{2-\alpha}(x),
$$

then the spectrum of our model can be described as

$\mathcal{P}_{\text {infOU }}=\frac{\zeta^{2} A^{2} x^{-\alpha}}{2 \pi(2-\alpha)}\left(\operatorname{Hyper}_{2 \mathrm{~F} 1}\left(1,1-\frac{\alpha}{2}, 2-\frac{\alpha}{2},-\left(\frac{\omega_{H}}{x}\right)^{2}\right)\left(\frac{\omega_{H}}{x}\right)^{2-\alpha}-\operatorname{Hyper}_{2 \mathrm{~F} 1}\left(1,1-\frac{\alpha}{2}, 2-\frac{\alpha}{2},-\left(\frac{\omega_{L}}{x}\right)^{2}\right)\left(\frac{\omega_{L}}{x}\right)^{2-\alpha}\right)$,

The hyper-geometric function $\operatorname{Hyper}_{2 \mathrm{~F} 1}(a, b, c, z)$ is defined as below,

$$
\operatorname{Hyper}_{2 \mathrm{~F} 1}(a, b, c, z)=\sum_{n=1}^{\infty} \frac{(a)_{n}(b)_{n}}{(c)_{n}} \frac{z^{n}}{n !}
$$

where $(q)_{n}$ is the Pochhammer symbol, which is defined by

$$
(q)_{n}= \begin{cases}1 & (n=0) \\ q(q+1) \ldots(q+n-1) & (n>0)\end{cases}
$$

This model is regarded as the infinite superpositions of OU process with scales from $\omega_{L}$ to $\omega_{H}$. Thus, it is more natural than Kelly et al. (2011)'s model which assumes that only finite discrete OU processes to contribute the variability. Since we can omit $M$ in the covariance function as shown in Equation 16, it does not require $M$ times computation in the covariance calculation, which is leading the faster calculation.

\subsection{Practical Implementation and Model Calculation}

We infer the model parameters by the following procedures. First, we estimate covariances for all measurement sets, by using each measurements and errors for sampling the data, to produce the covariance matrix, as described in 2.4. After calculating the likelihood by the covariance, we try to get posterior distribution of model parameters using MCMC sampler, and infer the best parameter values by getting medians of each parameter distributions. We 
use similar prior distribution to the prior used for the analysis by Kelly et al. (2011), but there is a difference in the range of $\alpha$, which is $-2<\alpha<0$ in Kelly et al. (2011)'s work. In our calculation for MAP estimation, for the prior distribution we assume the uniform distribution for $\alpha$ in the range of $-3<\alpha<3$. It is because the likelihood values are systematically larger than the case of Kelly et al. (2011)'s definition, for the objects which seem to have different PSDs inferred by DRW model. For $\zeta$ we use uniform prior in the range of $\zeta>0$, and also assume the uniform prior on $\omega_{L}$ and $\omega_{H}$, which satisfy the following relations, $\omega_{\min } \ll \omega_{L} \ll \omega_{\max }$, and $\omega_{L} \ll \omega_{H} \ll \omega_{\max }$. The upper and lower limits on the characteristic time scales, $\tau_{\max }=1 / \omega_{\min }$ and $\tau_{\min }=1 / \omega_{\max }$ are chosen to be $10^{5}$ and $10^{-2}$ days, respectively. We set the range of $\omega$ is about one order larger and smaller than the range of time intervals of the measurements. It is for avoiding the effects coming from boundary conditions in time for our analysis, and we only must see the parameter ranges which can be covered by the observations, if we want to interpret the resultant PSDs.

We use a part of JAVELIN package ${ }^{1}$ (Zu et al. 2011, 2013) for producing the light curve of the object with the given data points (usually 40 to 120 in our analysis), which is based on MCMC processing. In this package MCMC library emcee ${ }^{2}$ (Foreman-Mackey et al. 2012) is used for random sampling.

As the covariance function is an incomplete gamma function, we use the GNU Science Library for the faster calculation. As the JAVELIN package is written in python language, we use python as the language for our software development.

As the calculation for each object can be processed in parallel, we implemented the multi-process(parallel) calculation in our model analysis. Our model calculation takes about two to three minutes per object in single thread(depends on the number of data points), and we complete our model calculation in about five days for all 67,507 objects in our sample with 20 threads processing with CPU of Intel Xeon E5507(2.27GHz).

\section{DATA}

\subsection{SDSS Stripe 82 Photometric Catalog}

We use the gri photometric information from the Ivezic et al. (2007) variable source catalog, downloaded from the site $^{3}$. The catalog contains light curve information of 67,507 variable source candidates identified in Sloan Digital Sky Survey (SDSS) Stripe 82 area, based on SDSS Data Release 7 (DR7)(Abazajian et al. 2009). Their criteria for selecting the candidates are

1. unresolved source in imaging data, at least one band with photometric error below 0.05 mag

2. processing flags BRIGHT, SATUR, BLENDED, or EDGE are not set, meaning the measurement performed successfully

3. at least 10 observations in the $g$ and $r$ bands

4. the median $g$ band magnitude brighter than 20.5

5. root-mean-square scatter $>0.05$ magnitude and $\chi^{2}$ per degree of freedom larger than three in both $g$ and $r$ bands, which means the object is 'statistically' variable in brightness (see the discussions in Sesar et al. (2007) for the details)

This catalog is more extensive than the catalog of Sesar et al. (2007), because it includes both SDSS-I and SDSS-II, while the Sesar et al. (2007)'s catalog was based only on SDSS-I and therefore we decided to use this catalog for our research. Though this catalog also contains $u$ and $z$ band data, we did not use it for our study because of their lower signal-to-noise ratio(S/N) than those three (gri) bands data. The number of measurements in $r$ band is distributed in the range between $\sim 10$ to 140 , with maximum peak at around 60 .

\subsection{SDSS DR12 Spectroscopic Catalog}

We used SDSS Data Release 12(DR12) (Alam et al. 2015) data for identifying the spectroscopically confirmed variable source candidates in the photometric catalog mentioned in 3.1. On SDSS CasJobs system for DR12 data ${ }^{4}$, we used 'dbo.SpecPhoto' table to select the spectroscopically confirmed objects in the area of Stripe 82 . We specified

\footnotetext{
${ }^{1}$ http://www.astronomy.ohio-state.edu/ yingzu/codes.html\#javelin

2 http://dan.iel.fm/emcee/current/

${ }^{3} \mathrm{http}: / /$ www.astro.washington.edu/users/ivezic/sdss/catalogs/S82variables.html

${ }^{4}$ https://skyserver.sdss.org/CasJobs/
} 
the area by $|\delta|<1.266 \mathrm{deg}$. and RA in the range $20^{h} 34^{m}$ to $4^{h} 00^{m}$. The number of objects extracted from the table is 221,656, including 25,674 QSOs, 78,761 stars and 117,221 galaxies.

Spatial matching with the photometric catalog, based on $r$ band data, is performed for the coordinates(photora,photodec) in the table, by the criterion of the difference of the coordinates between photometric and spectroscopic catalogs within 1 arcsec. We identified 11,908 matched objects and they are 8,105 QSOs, 3,379 stars and 424 galaxies, based on the spectroscopic classification on the table 'dbo.SpecPhoto', described as 'QSO', 'STAR' and 'GALAXY' in column 'Class', respectively. There are 17 sources with multiple sources in the searched area, and we selected the nearest one as the counterpart in the cases.

It is noted that most spectroscopically confirmed objects in the photometric catalog have measurements over 40 . For the validation of selection ability of QSOs from stars based only on their variability, we need a sample with enough number of photometric measurements and spectroscopic identifications. Thus we decide to use the objects which are spectroscopically confirmed and have measurements number over 40 with good accuracy, with the error of each measurement less than 0.05 magnitude, for our test of QSO selection, which hereafter is called a 'good sample' hereafter, although we calculate the model parameters on all objects to investigate the effects of measurement numbers on our analysis. The total number of objects in the 'good sample' is 9,855, with 7,714 QSOs and 2,141 stars.

One important features that affects the time series analysis using irregularly and sparsely sampled data is the cadence of each object and its similarity. We consider that the cadences in the sample are similar to each other, if the distribution of the sets of time intervals for each object, which is the histogram of time intervals, are similar for whole sample. To check the cadence of the 'good sample', we calculated time intervals of multiple measurements for each object in the sample, and produced the histogram as shown in Figure 1(a). We used 100 bins equally divided between -1 to 4 on log-scaled time intervals in days, and stacked all the time intervals of each object to produce a stacked cadence template histogram. It is clear that the minimum time intervals are less than $\sim 1$ day, and $\sim 10$ years at the maximum, and we can obtain a certain level of information on variability about such a time-scale range.

For investigating the similarity of the cadences of our sample, we used the Bhattacharyya coefficient(hereafter BC)(Bhattacharyya 1943), which is frequently used for checking the similarity of two histograms. The definition of BC can be written as

$$
B C(p, q)=\sum_{i=1}^{n} \sqrt{p_{i} q_{i}}
$$

where $p_{i}$ and $q_{i}$ are normalized count in $i$-th bin of two histograms. In this case they are histograms for stacked cadence template and for single object, respectively. If both histograms are the same, $B C$ value will be one, and decrease as dissimilarity increases.

The distribution of $B C$ s for the 'good sample', shown in Figure 1(b), means that the cadences are very similar, as the most of all objects show the values more than 0.95. This feature of the data set is suitable for detecting different types of intrinsic variability as we can decrease the effect coming from irregular and sparse sampling. It should be noted that the results are the same for histogram with 1 day bin size, which means that the cadence greater than 1 day long are very similar for whole sample.

The similarity of the cadences are easily imagined because SDSS Stripe 82 data are taken through the time-delay-andintegrate (TDI) scanning mode(Gunn et al. 1998), which enable homegeneous scanning of the sky in each observation night.

As is already mentioned, we cannot investigate the details of intrinsic variability since the data suffer from irregular and sparse time sampling. Considering the similarity of the cadence, however, the data provide enough information for detecting the relative differences of the photometric variations, and they can be powerful to separate QSOs from stars. It is meaning that the effects coming from the irregular and sparse time sampling will emerge in the same way to the results for all sample objects and intrinsic features can be distinguished relatively.

\section{RESULTS}

\subsection{Fitted Light Curves and Derived Parameters}

By applying our model to the 67,507 variable sources, we derived four parameters $\left(\omega_{H}, \omega_{L}, \zeta, \alpha\right)$ for all sources. These parameters are estimated by taking the median of posterior distributions, and lower and higher errors are estimated by getting the difference between the median and $16 \%$ or $84 \%$ quantile values, respectively. We calculated them on $g, r$, and $i$ band data independently. As the ability of distinguishment of QSOs from stars are very similar among the data-set on three filters, we limit our description only on $r$ band data in the following. 


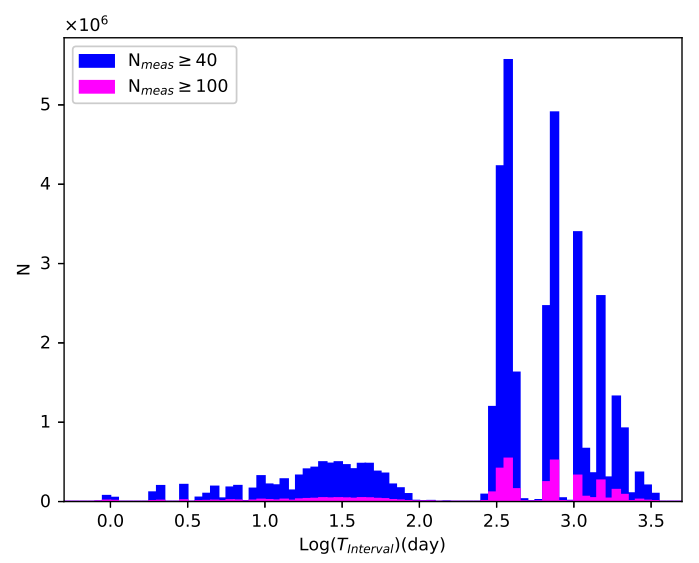

(a)

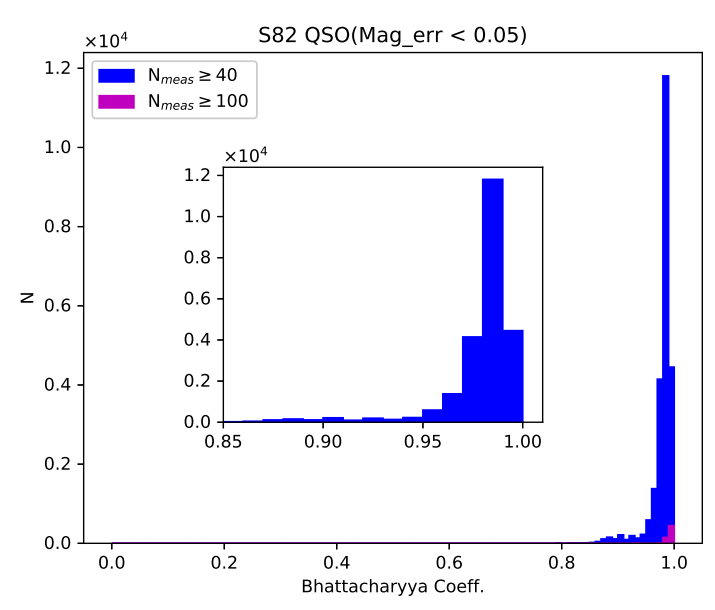

(b)

Figure 1. (a)The histogram which shows the 'stacked' cadence of photometric data of the 'good sample'. The time intervals are in log-scale. Blue histogram shows for the objects with measurements $\geq 40$, and magenta for those with $\geq 100$ measurements. It is clear that the cadence is not heavily dependent on the number of measurements. (b)The histogram of $B C$ s for all objects in the 'good sample'. The zoom-up around $0.85<B C<1.0$ is shown in the inner panel.

In Figures 2 and 3, we show the inferred light curves for one of the spectroscopically confirmed QSOs and stars, respectively. These figures also show their posterior distributions of inferred parameters in histograms, on the data sets of posteriors in contours, and their inferred power spectral densities (PSDs), which will be described on the details in 4.2. The shaded part of the light curves show the range of $68 \%$ confidence level of our calculation based on MCMC, and the solid lines mean the inferred light curves, derived from median of the 20,000 chain results.

On the most spectroscopically confirmed QSO, we can get well fitted inferred light curves, which are characterized by variation with long time scale(e.g. small frequency) (Figure 2(a)). They are characterized by small $\omega_{L}$ and $\omega_{H}$.

On the other hand, we cannot have a good fit on stars light curve, with flat light curves without tracing the measurements, as shown in Figure 3(a). It is however not the failure of our model fitting because our model uses only a type of PSD assuming the QSO/AGN like variability. In fact the inferred PSD has larger $\zeta$ compared to QSO/AGN, and flat shape in small $\omega$ range which means the variability is like white noise. As the most variable stars are periodically variating their brightness, it is consistent to the the fact. Most importantly inferred parameters are good for the purpose of separating stars from QSOs in the parameters space, as shown in next coming sections.

There are also 37 spectroscopically confirmed QSO in the 'good sample' without successful light curve fits. They cannot be distinguished from most stars in optical variability using our model. On the other hand, we identify 199 QSOlike light curves on the objects classified as star on the SDSS spectra. We investigated carefully their optical spectra by visual inspection and determined 16 of them are possibly misidentified as 'STAR'. Many misidentified objects have relatively weak emission lines in their spectra and are possibly categorized as weak emission line QSOs(Plotkin et al. 2010).

We should note that the inferred parameter $\omega_{L}$ for many QSOs are less than $\sim 1 / 4000$ days $^{-1}$, the maximum duration limit of the data( $\sim 10$ years). It is because we set the prior of $\omega_{L}$ as uniform distribution in the range of $-5<\log \omega_{L}<2$. The values are thus the results of fitting our model to the data with time-scale shorter than $\sim 4,000$ days, and $\omega_{L}$ smaller than $4000^{-1}$ day $^{-1}$ is only the extrapolation based on our model. We should emphasize here that the values of $\omega_{L}$ are meaningful, as our purpose is only to identify QSOs, not to infer PSDs correctly, by using a model with approximate PSD expression for describing variability of QSOs. We also mention briefly the interpretation of the inferred PSDs in 6.3.

For confirming the consistency of our model with Kelly et al. (2011)'s finite OU mixture model, we checked the value of information criteria on the results. We use BIC(Bayesian Information Criterion)(Schwarz 1978) for this check. It is defined as the following equation, 


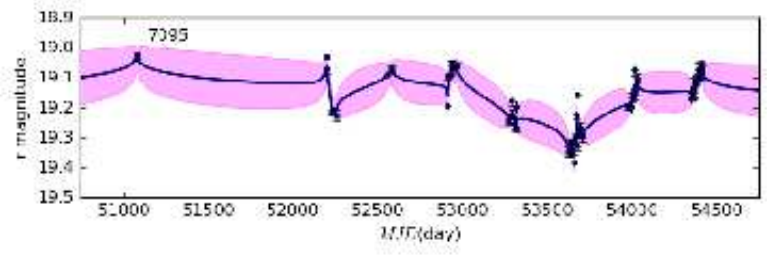

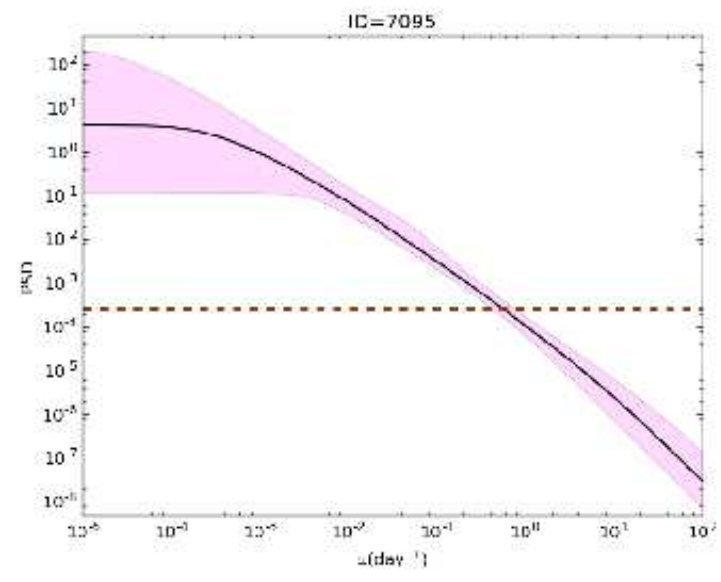

(a)

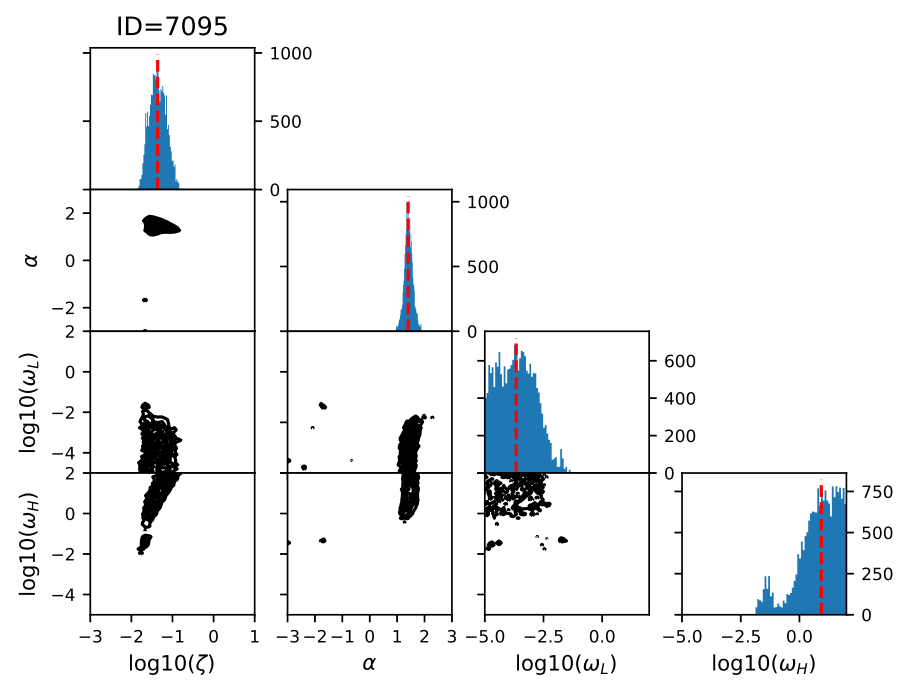

(b)

Figure 2. One result of our model calculation for a QSO(ID number 7095). (a)Upper left: It shows the light curve with shaded area which presents the $68 \%$ confidence range for the inferred light curve. The results of photometric measurements are shown by filled circle with error bars. Lower left: This shows the inferred PSD with $90 \%$ confidence range with shaded area. The horizontal dashed lines show the median(red) and average(green) values of errors on photometric measurements. The vertical blue dashed lines show inferred $\omega_{L}$ and $\omega_{H}$, respectively. (b)Pair plot which shows the posterior distributions of our model parameters, $\log \zeta, \alpha, \log \omega_{L}$, and $\log \omega_{H}$ from left to right, respectively. The histograms show the posterior distributions of each parameters, with median values indicated by the red dashed lines. Contour maps showing the distributions of the posteriors on each set of our model parameters $\left(\zeta, \alpha, \omega_{L}, \omega_{H}\right)$ about the object in MCMC chains.

$$
B I C=k \ln (n)-2 \ln (L)
$$

where $L$ is the maximum value of likelihood function, $n$ is the number of measurements, and $k$ is the number of estimated parameters in the model.

Information criteria are a common and useful mechanism for ranking a set of models. In time series analysis BIC and/or AIC(Akaike Information Criterion)(Akaike 1973) are used so frequently, which is based on the maximumlikelihood estimate of the parameters. The BIC/AIC provide estimates of the relative information lost in using a model to represent the underlying process that generated the data. As we used Bayesian inference for our analysis, we use BIC for the check.

In Figure 4, we show 3 samples of BICs distributions on $M$ about spectroscopically confirmed QSOs. There are various tendencies on the distribution, however we can see our infinite OU mixture model provides the same level of the goodness of the fit compared to Kelly et al. (2011)'s finite OU mixture model with large mixture numbers. It is also consistent with the suggestion by Kelly et al. (2011) that the number of mixed OU processes should be larger than 30 to describe observed AGN's light curves sufficiently. We should note here that the numbers of mixed OU processes $(M)$ 


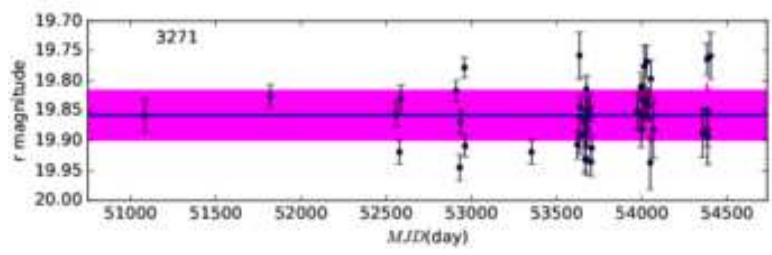

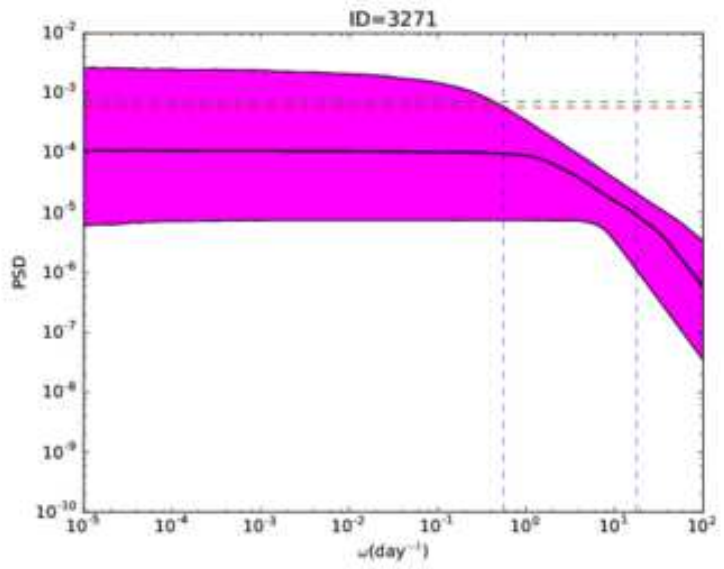

(a)

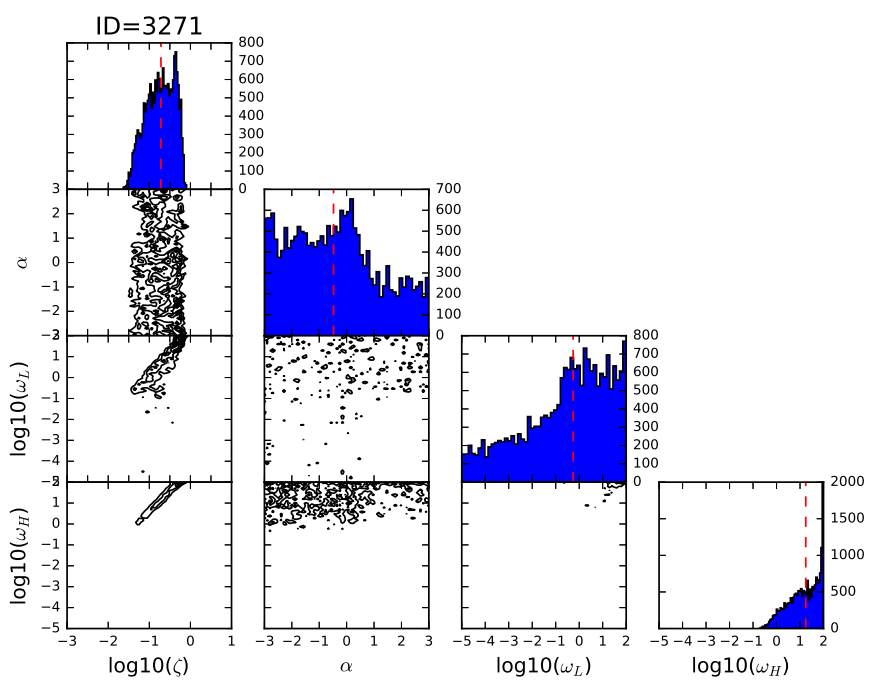

(b)

Figure 3. The same one of Figure 2 for a star(ID number 3271).

which provide the best BIC on mixed OU model for the spectroscipically confirmed QSOs in 'good sample' change object to object, although most of them are $M=32,64,128$ or $\infty$.

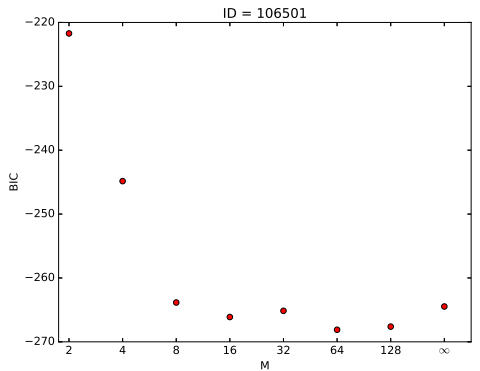

(a)

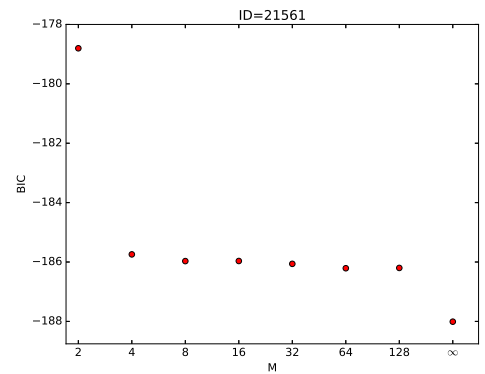

(b)

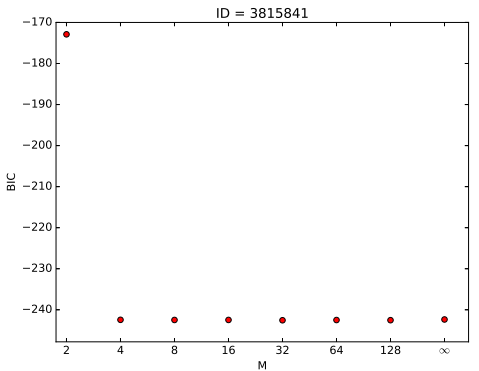

(c)

Figure 4. BICs dependencies on the number of mixed OU processes about 3 sample objects. BIC on $M \rightarrow \infty$ show the comparable values to those for $M>30$.

For checking the flexibility of our model to AGN/QSO variability, we show the covariance functions in Figure 5(a). The crosses are covariance values calculated by the observation data set. We select 5 objects which are representative of our model parameter space as shown in Figure 5(b). We named them as 'Long', 'Middle', 'Short', 'DblBend' and 'PowerLaw'.

'Long', 'Middle' and 'Short' represent the difference of variability time scales, and they show smaller difference between $\omega_{H}$ and $\omega_{L}$. 'DblBend' and 'PowerLaw' represents the shapes of PSD with double-bending and power-law which have much difference between $\omega_{H}$ and $\omega_{L}$. The difference between 'DblBend' and 'PowerLaw' is whether $\omega_{H}$ is lower or higher than 1 day $^{-1}$, which corresponds to the minimum of time intervals of the data. 
It is clear that the covariance functions can describe well the difference of variabilities by changing their height and shape, especially in short time scale range.

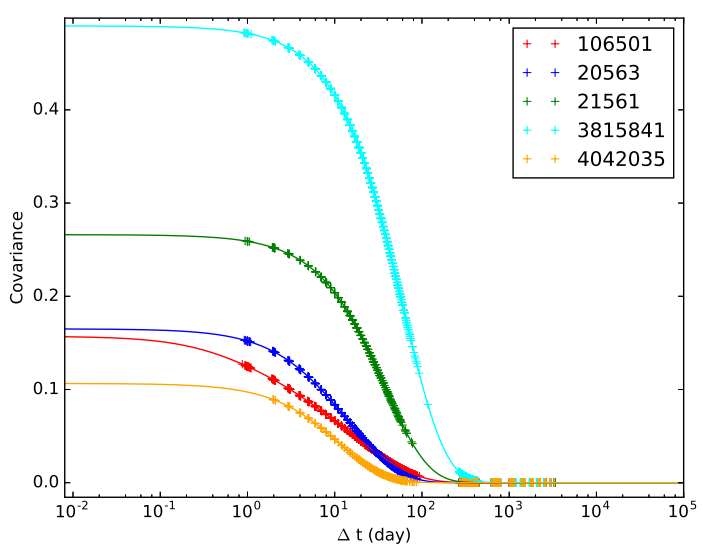

(a)

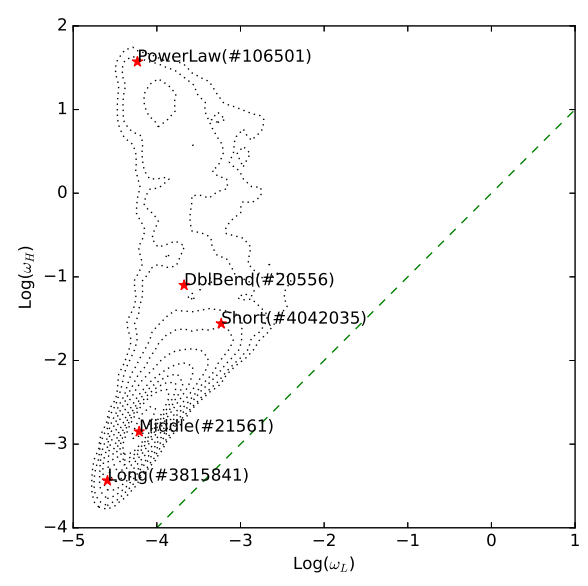

(b)

Figure 5. (a)Covariance functions for 5 sample objects with different types of variabilities. (b)The contours with dotted lines show the distribution of spectroscopically confirmed QSOs in our 'good sample' on $\omega_{L}-\omega_{H}$ plane. The types of variabilities are shown with ID number of the representative objects.

\subsection{PSD Calculation and Derived Parameters}

For investigating the details of the variability, we derive the power spectral density (PSD) for each object. The method to calculate the PSD is described in 2.4, and PSDs for a sample QSO/AGN and star are shown in Figure 2 and Figure 3. We can see some typical features in PSDs for QSOs and stars. For example, many QSOs show the PSDs with smoothly changing slopes, the turning points appears at the border of white noise (slope $\beta=0$ ) and red noise $(\beta=-2)$, when we express the PSD $\propto \omega^{\beta}$. On the other hand, stars' PSD inferred by our model has a bending point at larger $\omega$ range $(\log \omega>0)$, with flat spectra in smaller $\omega$ range, which is consistent to white noise. Since our model originally needs four parameters for describing PSD for the variability of QSOs, it is not easy to visualize the behavior of inferred PSDs among different celestial objects, especially QSOs and stars. We therefore introduce the parameters, which show the typical frequency and height of PSDs. As the PSD derived from our model has the features that the slope will be asymptotic to 0 in $\omega \rightarrow-\infty$, and to -2 in $\omega \rightarrow \infty$, we define two asymptotic lines for the PSD and derive the $(\omega, P(\omega))$ at the crossing point of these lines, as $\left(\omega_{c}, P_{c}\right)$, and then we try to describe our model with three parameters $\omega_{c}, P_{c}$ and $\alpha$ (Figure 6). It means that $\omega_{c}, P_{c}$ and $\alpha$ are expressing the features of PSDs, which are corresponding to the absolute position of typical time scale, the amplitude, and difference of maxmum and minimum time scales.

We define the feature point on the power spectrum derived from our model as the crossing point of the asymptotes in the cases of $\omega \rightarrow 0$ and $\omega \rightarrow \infty$. When $\omega \rightarrow 0$, the power spectrum can be written as below.

$$
\frac{\zeta^{2} A^{2}}{2 \pi} \int_{\omega_{L}}^{\omega_{H}} \frac{\omega^{1-\alpha}}{\omega^{2}} d \omega=\frac{\zeta^{2} A^{2}}{2 \pi} \int_{\omega_{L}}^{\omega_{H}} \omega^{-1-\alpha} d \omega=\frac{\zeta^{2} A^{2}}{2 \pi} \frac{\omega_{H}^{-\alpha}-\omega_{L}^{-\alpha}}{-\alpha},
$$

On the other hand in case of $\omega \rightarrow \infty$, we can derive the spectrum as below.

$$
\frac{\zeta^{2} A^{2}}{2 \pi} \int_{\omega_{L}}^{\omega_{H}} \frac{\omega^{1-\alpha}}{x^{2}} d \omega=\frac{\zeta^{2} A^{2}}{2 \pi} \frac{1}{x^{2}} \int_{\omega_{L}}^{\omega_{H}} \omega^{1-\alpha} d \omega=\frac{\zeta^{2} A^{2}}{2 \pi} \frac{1}{x^{2}} \frac{\omega_{H}^{2-\alpha}-\omega_{L}^{2-\alpha}}{2-\alpha},
$$

By satisfying the both formulas simultaneously, the coordinate of crossing point is derived as

$$
x=\sqrt{\frac{-\alpha}{\omega_{H}^{-\alpha}-\omega_{L}^{-\alpha}} \frac{\omega_{H}^{2-\alpha}-\omega_{L}^{2-\alpha}}{2-\alpha}} \equiv \omega_{c}
$$




$$
y=\frac{\zeta^{2}}{2 \pi} \frac{2-\alpha}{\omega_{H}^{2-\alpha}-\omega_{L}^{2-\alpha}} \frac{\omega_{H}^{-\alpha}-\omega_{L}^{-\alpha}}{-\alpha} \equiv P_{c}
$$

, and we can define the point as the feature point.

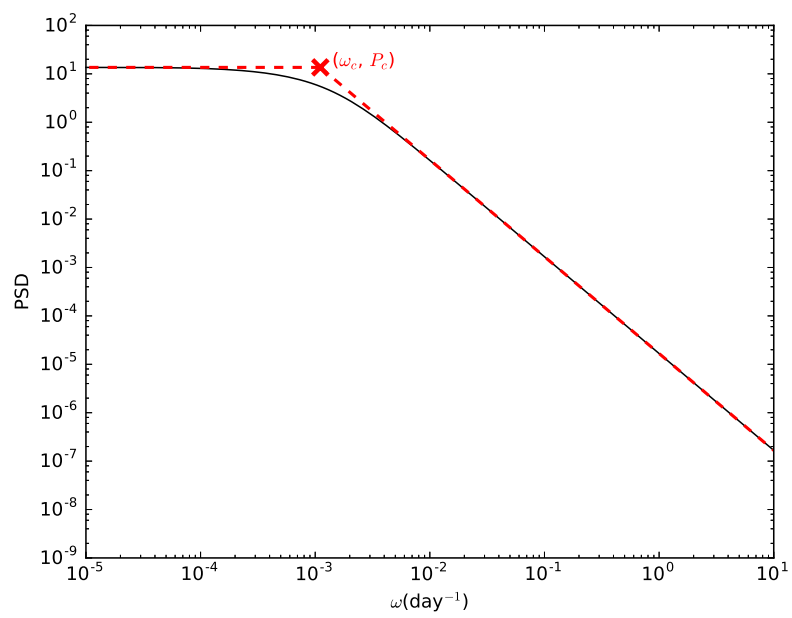

Figure 6. The schematic figure showing how the values of PSD's parameters $\omega_{c}$ and $P_{c}$ are derived.

We show the distribution of these three parameters $\left(\alpha, \log \omega_{c}\right.$ and $\left.\log P_{c}\right)$ on 67,507 variable sources by the 3 - $\mathrm{d}$ contour plot in Figure $7(\mathrm{a})$, which indicates at least three clusters and two extensions in the distribution. The clusters are characterized by large $P_{c}$ and small $\omega_{c}$ (Group1), small $P_{c}$ and large $\omega_{c}$ (Group2), and small one neighboring to Group2 with smaller $\alpha$ (Group3). There are two extensions, one is the narrow one connecting Group1 and Group2(Ext1), and another is extending to smaller $\alpha$ direction(Ext2).

For checking the correspondence of the objects types (especially on QSOs and stars) to these structures in Figure 7(a), we plot the same figure only for the objects with spectroscopic confirmations as 'QSO' or 'STAR' in Figure 7(b). It is clear that the gap between Group1 and Group2 is clear in Figure 7(b), which is not visible in Figure 7(a). Additionally we can barely see Group3 and Ext2, and can see Ext1 in the range of smaller $\omega$ range.

It should be noted that the main contributors of these disappering structures are objects with small numbers of measurements, which suffer from the noisy inference of the parameters. We confirm that the objects labeled 'GALAXY' in the spectroscopic catalog of SDSS DR12 also belong to any of these structures.

We also plot these three parameters of each spectroscopically confirmed QSOs and stars on the same parameter space in Figure 8, to see the correlation between spectroscopic classification and variability features. These figures show that the majority of QSOs and stars belong to Group1 and Group2, respectively, and Group3 consists of stars. Stars are also the main contributors of two extensions.

On the QSOs and stars in 'good sample', the effect caused by a small number of photometric measurements is reduced, and we can see more clearer separation of QSOs and stars in the parameter space. It means that distinguishing QSOs from stars is probably possible in very high identification rate, by using our model parameters, as probed by many previous similar works based only on optical variability (for examples, Kozlowski et al. (2010a); MacLeod et al. (2010, 2011); Butler \& Bloom (2011); Andrae et al. (2013)). The accuracy of the distinguishment should be higher in the case the number of photometric measurements increases.

Another common clear feature is that the $\omega_{c}$ is larger the $P_{c}$ is smaller in QSOs(Group1). This means that the power of variability is higher the longer the damping time scale is. This is the same feature revealed by DRW model analysis(MacLeod et al. 2011).

\section{CLASSIFICATION AND IDENTIFICATION RATE OF OUR MODEL}




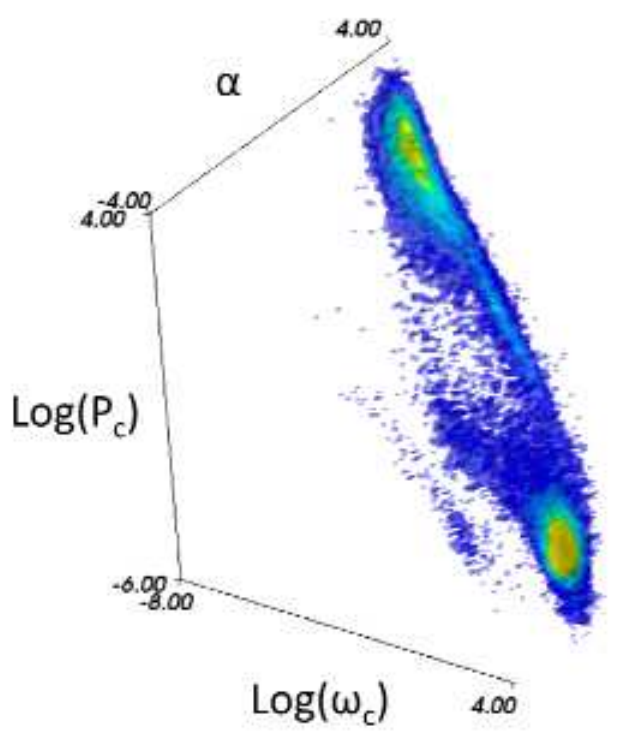

(a)

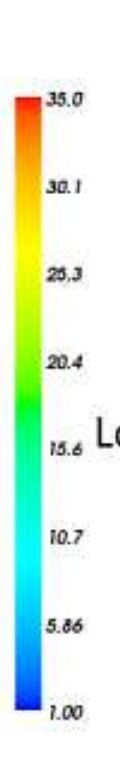

. 

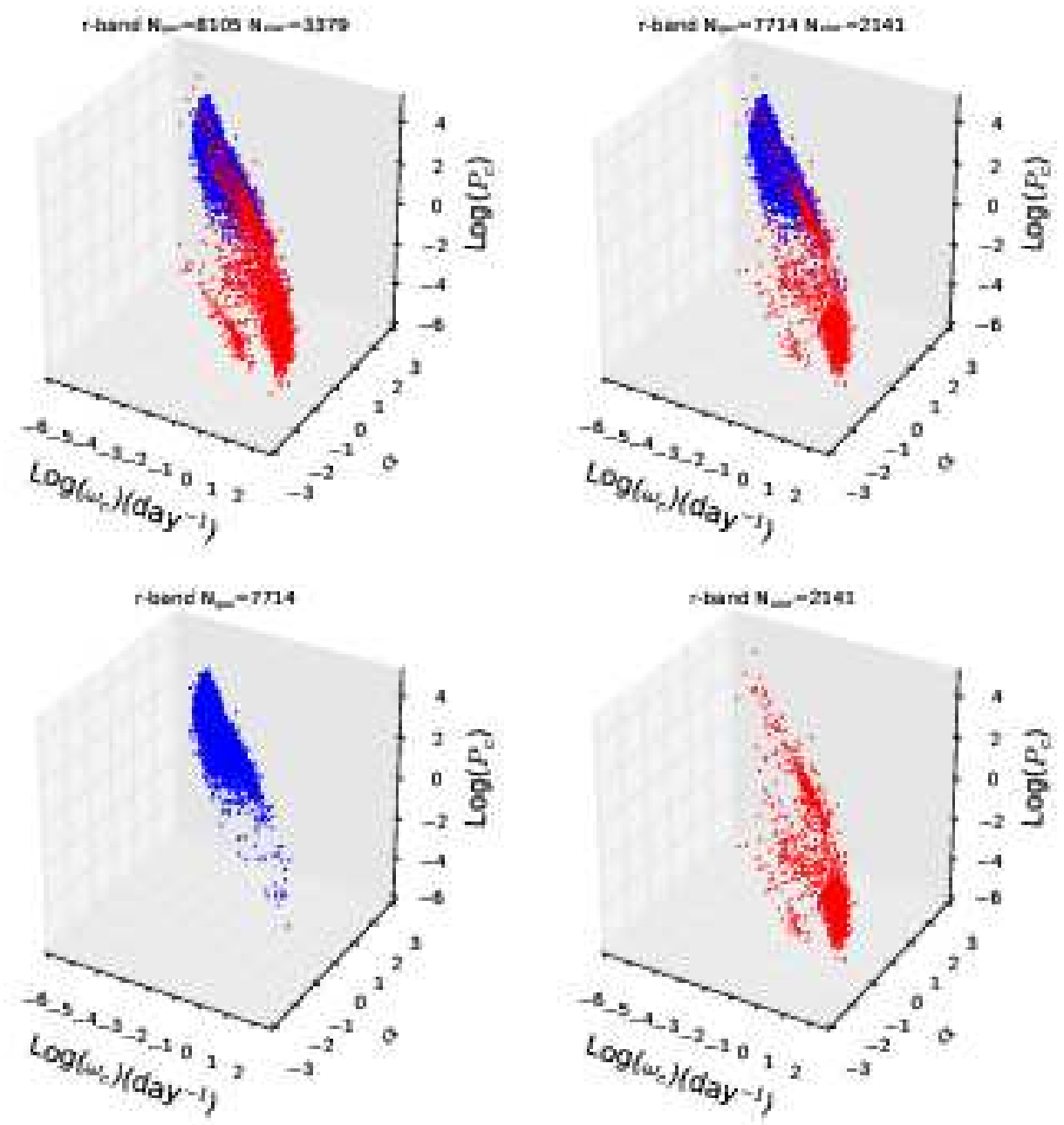

Figure 8. Distribution of spectroscopically identified QSOs (blue) and stars(red) in derived parameters space $\left(\omega_{c}, \alpha, P_{c}\right)$. Upper left figure shows all sample data (8,105 QSOs and 3,379 stars). Upper right figure is based on the 'good sample' data. There are 7,714 QSOs and 2,141 stars. Lower left and right figures show only QSOs and stars,respectively.

using the 'good sample'. Since we want to clarify the capability of optical variability to select QSOs, we constrain the discussions only to the results based on the 'good sample' hereafter if there is no notification.

It is clear that the identification rates based on our optical variability modeling are very high, completeness is at about $97-99 \%$ for all parameter sets, purities at $98-99 \%, 93-95 \%$ in accuracies, and $89-92 \%$ in recall, respectively. There are very small differences among the identification rates by the different parameter sets. In completeness, the highest value is achieved by 'Inf4', 'Inf3oz', and 'Inf2zl' data set with $99.3 \%$. 'Inf4' also provides the highest in purity $(99.0 \%)$, in accuracy (95.3\%), and 'Inf3cr' in recall(92.2\%). It is noted that the rates are slightly decreased by adding the photometrically less measured sample (not 'good sample'), as represented by the data sets 'InfAll4' and 'InfAll3cr' in Table 1, which are based on the sample of all spectroscopically confirmed 8,105 QSOs and 3,379 stars.

By 1,000 trials of the classification using linear SVM, we can calculate the identification rates of our classification on each source, by counting up the numbers of successful classification on sources in the test sample. As the test data are randomly selected in each trial, all QSOs and stars in the 'good sample' are selected into test data homogeneously. Therefore we can calculate the identification rate for the whole sample without systematic biases. The range on the 


\begin{tabular}{|c|c|c|c|}
\hline \multirow{2}{*}{\multicolumn{2}{|c|}{ Variability Spectroscopy }} & \multirow{2}{*}{$\begin{array}{l}\text { QSO in spectroscopy } \\
\text { True }\end{array}$} & \multirow{2}{*}{$\begin{array}{l}\text { Star in spectroscopy } \\
\text { False }\end{array}$} \\
\hline & & & \\
\hline QSO in variability & Positive & TP (True Positive) & FP(False Positive) \\
\hline Star i & Negat & & e) \\
\hline Completeness & $\frac{T P}{T P+T N}$ & \multicolumn{2}{|r|}{$\begin{array}{r}T P+T N \\
\end{array}$} \\
\hline neco & $\frac{T P}{D+F}$ & \multicolumn{2}{|c|}{$\operatorname{Precision}($ Purity $)=\frac{T P}{T P+F P}$} \\
\hline
\end{tabular}

Figure 9. The definitions of values for describing identification rates used in our analysis.

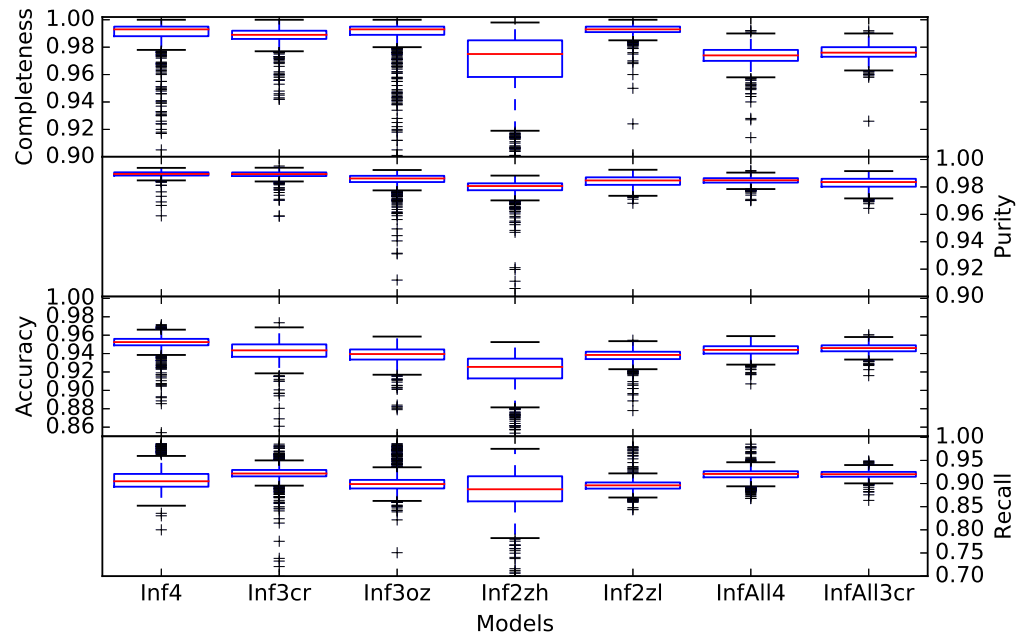

Figure 10. Box plot of the identification rates of our models. This figure shows the results on completeness, purity, accuracy, and average precision on $r$ band data, respectively. The box extends from the lower to upper quartile values of the data (blue lines), with red lines at the median. The whiskers extend from the box to show the range of the data, Q3 $+1.5 \times \mathrm{IQR}$. IQR is inter-quartile value (Q3 - Q1), when Q1 and Q3 are the 1st and 3rd quartile values. Flier points are for those past the end of the whiskers.

numbers of selection to test data is 120 to 190 , with median value of $\sim 155$, for QSOs and 520 to 600 , with median value of $\sim 560$, for stars. In Figure 11, we show the distribution of QSOs and stars in three-dimensional parameter $(\alpha$, $\left.\log \omega_{c}, \log P_{c}\right)$ space with their success rates, based on the results from our model('Inf4'). We can see the well-defined borders of QSOs and stars in our classification.

\section{DISCUSSIONS}

\subsection{Comparison with other models on identification rates}

For confirming the performance of our model in QSO selection, we compare the identification rates (discrimination ability of 'QSO' and 'STAR') to several models. The models we use for the comparison are Butler's 
Table 1. Comparison of the Identification Rates on the sets of our model parameters

\begin{tabular}{ll|lrlc}
\hline \hline Abbreviation & Parameters & Completeness & Precision(Purity) & Accuracy & Recall \\
\multicolumn{7}{l}{} \\
\multicolumn{7}{l}{ Inf4 } & $\omega_{L}, \omega_{H}, \zeta, \alpha$ & $0.993_{-0.002}^{+0.005}$ & $0.990_{-0.001}^{+0.001}$ & $0.956_{-0.004}^{+0.004}$ & $0.905_{-0.016}^{+0.012}$ \\
Inf3cr & $\omega_{c}, P_{c}, \alpha$ & $0.989_{-0.003}^{+0.003}$ & $0.989_{-0.001}^{+0.002}$ & $0.944_{-0.007}^{+0.007}$ & $0.922_{-0.008}^{+0.006}$ \\
Inf3oz & $\omega_{L}, \omega_{H}, \zeta$ & $0.993_{-0.003}^{+0.005}$ & $0.986_{-0.002}^{+0.003}$ & $0.940_{-0.005}^{+0.006}$ & $0.899_{-0.009}^{+0.010}$ \\
Inf2zh & $\omega_{H}, \zeta$ & $0.974_{-0.010}^{+0.016}$ & $0.981_{-0.002}^{+0.003}$ & $0.926_{-0.009}^{+0.013}$ & $0.887_{-0.028}^{+0.026}$ \\
Inf2zl & $\omega_{L}, \zeta$ & $0.993_{-0.002}^{+0.002}$ & $0.985_{-0.002}^{+0.003}$ & $0.939_{-0.004}^{+0.005}$ & $0.896_{-0.006}^{+0.007}$ \\
& & & & & \\
InfAll4 & $\omega_{L}, \omega_{H}, \zeta, \alpha$ & $0.974_{-0.004}^{+0.004}$ & $0.985_{-0.002}^{+0.002}$ & $0.944_{-0.004}^{+0.004}$ & $0.921_{-0.006}^{+0.007}$ \\
InfAll3cr & $\omega_{c}, P_{c}, \alpha$ & $0.976_{-0.004}^{+0.003}$ & $0.984_{-0.002}^{+0.003}$ & $0.946_{-0.003}^{+0.004}$ & $0.920_{-0.005}^{+0.005}$ \\
\hline
\end{tabular}
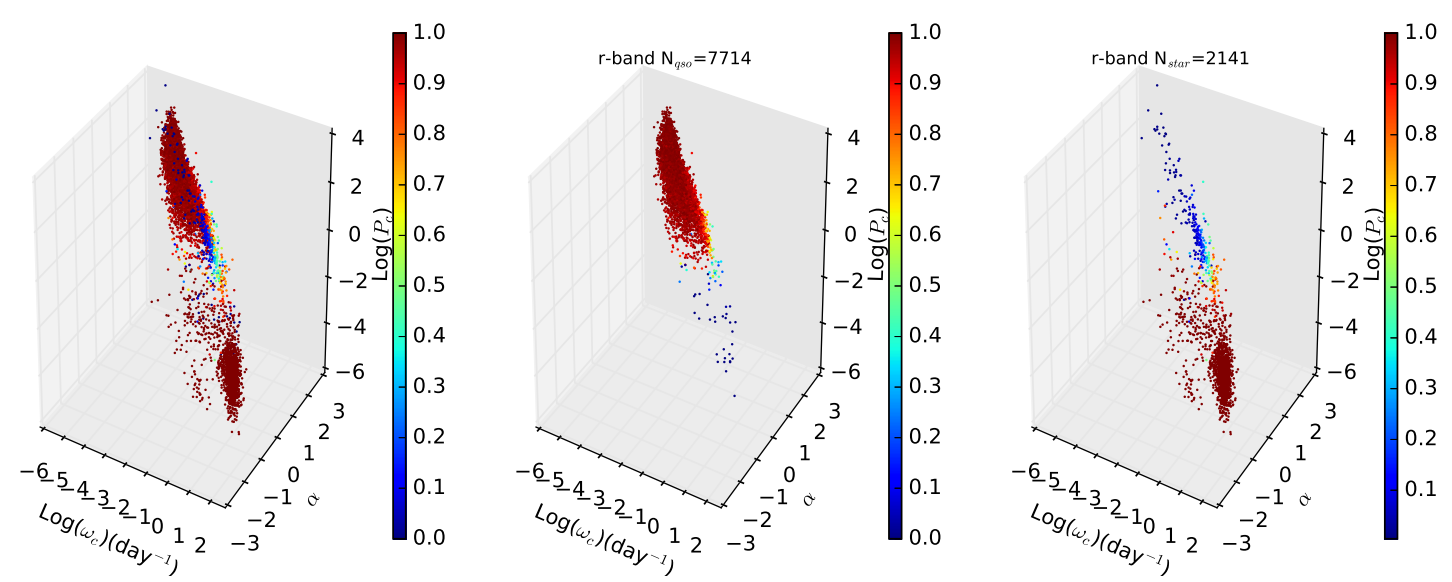

Figure 11. The same as Figure 8, but with their success rates in the classification using their optical variability with our model analysis. The left one is showing the distribution of spectroscopically confirmed QSOs and stars, and the middle and right plots are only for QSOs and stars, respectively. The colors show the success rates of each source. The color bar presents the success rates for each spectroscopically confirmed QSO and star.

model(Butler \& Bloom 2011), Zu's single OU model(Zu et al. 2011, 2013), and Kelly's OU mixture models(Kelly et al. 2011).

Butler \& Bloom (2011) use the DRW model by Kelly et al. (2009) and implement the two parameters $\chi_{q s o}^{2} / \nu$ and $\chi_{\text {false }}^{2} / \nu$ for well separating QSOs and stars in the parameter space, and it is believed to be one of the most powerful tools for selecting QSOs. We use their software released on their web page ${ }^{6}$. For computation using a single OU process, we use the JAVELIN package developed and maintained by Zu et al. (2011, 2013). Their program outputs $\tau$ and $\sigma(\zeta$ in 2.2) and well separates QSOs and stars in two-dimensional parameter space. We develop the program for calculation based on Kelly's OU mixture model(Kelly et al. 2011). We use the same MCMC program with our model calculation and we investigate in the cases of mixture number of OU process $M$ are equal to 2, 4, 8, 16, 32, 64 and 128. As the time consumed for the OU mixture model calculation is too long to calculate on all sample objects, we limit the calculation only to those with spectroscopic confirmation(11,908 objects). On the other hand we calculate

\footnotetext{
${ }^{6}$ http://butler.lab.asu.edu/qso_selection/index.html
} 
67,507 objects for Butler and single OU models. The distributions of QSOs and stars in the parameter spaces of each model are shown in Figure 12, and they are showing good performance on the separating QSOs from stars.

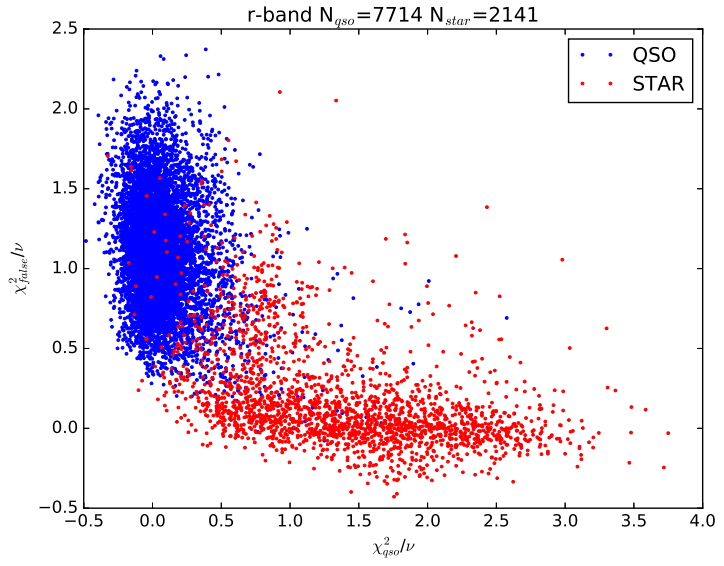

(a)

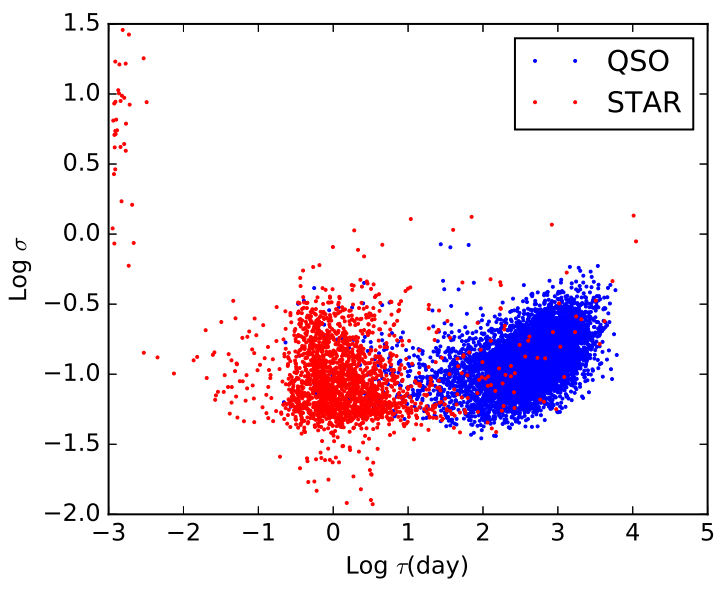

(b)

Figure 12. (a)Distribution of model parameters of spectroscopically confirmed QSO(blue) and STAR(red) for the model of Butler \& Bloom (2011). (b)That of QSOs and STARs parameters $(\tau$ and $\sigma$ ) for the model of Zu et al. (2011). The number of plotted QSO and STAR are 7,714 and 2,141, respectively.

The classifications are performed in the same manner as for our model, and we derive the same values for estimating their discrimination abilities. Per each sample, we have $\log \tau, \log \sigma$ for single OU model, $\chi_{q s o}^{2} / \nu, \chi_{\text {false }}^{2} \nu$ for Butler's model and $\log \omega_{L}, \log \omega_{H}, \alpha, \log \zeta, \log \omega_{c}, \log P_{c}$ for Kelly's mixture model. We then apply the classifiers to the subsets of these parameters and determine whether the samples are QSOs or not.

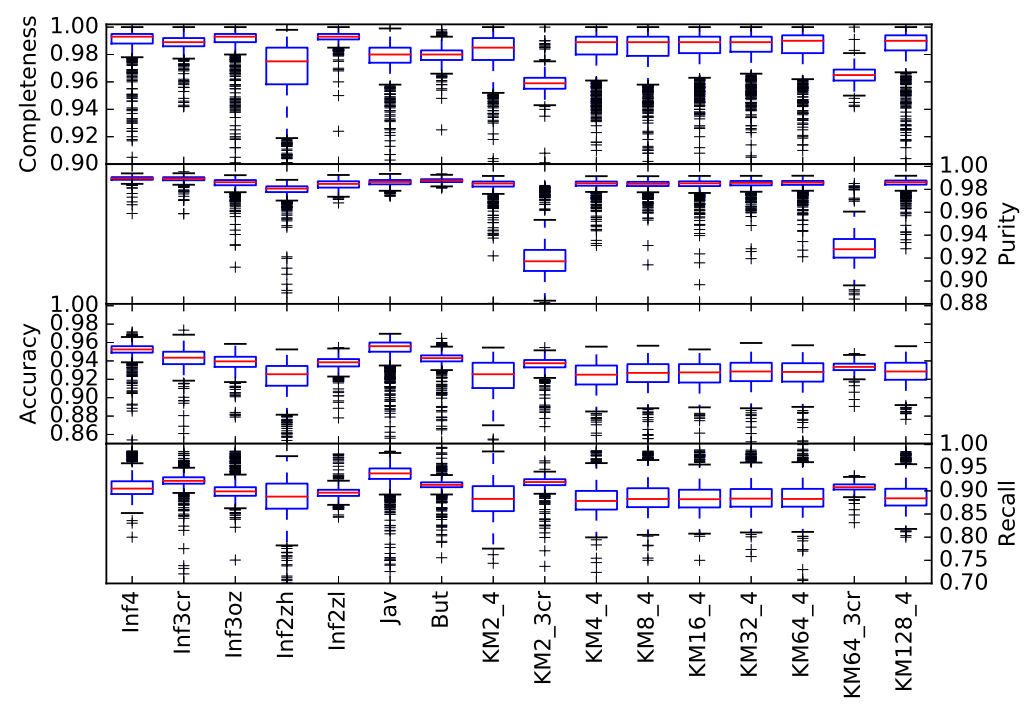

Figure 13. Box plot for comparisons of identification rates on the different models in the same manner as Figure 10. 
Table 2. Comparison of Identification Rates among models

\begin{tabular}{|c|c|c|c|c|c|c|}
\hline Model & Abbreviation & Parameters & Completeness & Precision(Purity) & Accuracy & Recall \\
\hline \multirow[t]{5}{*}{ Our Model } & $\operatorname{Inf} 4$ & $\omega_{L}, \omega_{H}, \zeta, \alpha$ & $0.993_{-0.002}^{+0.005}$ & $0.990_{-0.001}^{+0.001}$ & $0.956_{-0.004}^{+0.004}$ & $0.905_{-0.016}^{+0.012}$ \\
\hline & Inf3cr & $\omega_{c}, P_{c}, \alpha$ & $0.989_{-0.003}^{+0.003}$ & $0.989_{-0.001}^{+0.002}$ & $0.944_{-0.007}^{+0.007}$ & $0.922_{-0.008}^{+0.006}$ \\
\hline & Inf3oz & $\omega_{L}, \omega_{H}, \zeta$ & $0.993_{-0.003}^{+0.005}$ & $0.986_{-0.002}^{+0.003}$ & $0.940_{-0.005}^{+0.006}$ & $0.899_{-0.009}^{+0.010}$ \\
\hline & Inf2zh & $\omega_{H}, \zeta$ & $0.974_{-0.010}^{+0.016}$ & $0.981_{-0.002}^{+0.003}$ & $0.926_{-0.009}^{+0.013}$ & $0.887_{-0.028}^{+0.026}$ \\
\hline & Inf2zl & $\omega_{L}, \zeta$ & $0.993_{-0.002}^{+0.002}$ & $0.985_{-0.002}^{+0.003}$ & $0.939_{-0.004}^{+0.005}$ & $0.896_{-0.006}^{+0.007}$ \\
\hline JAVELIN & $\mathrm{Jav}$ & $\tau, \sigma$ & $0.980_{-0.005}^{+0.007}$ & $0.986_{-0.002}^{+0.002}$ & $0.956_{-0.004}^{+0.006}$ & $0.938_{-0.011}^{+0.012}$ \\
\hline BUTLER & But & $\chi_{q s o}^{2} / \nu, \chi_{\text {false }}^{2} / \nu$ & $0.980_{-0.003}^{+0.004}$ & $0.988_{-0.001}^{+0.001}$ & $0.943_{-0.003}^{+0.004}$ & $0.913_{-0.006}^{+0.005}$ \\
\hline $\operatorname{Mix}(M=2)$ & KM2_4 & $\omega_{L}, \omega_{H}, \zeta, \alpha$ & $0.985_{-0.007}^{+0.010}$ & $0.985_{-0.002}^{+0.003}$ & $0.926_{-0.013}^{+0.015}$ & $0.882_{-0.027}^{+0.026}$ \\
\hline $\operatorname{Mix}(M=4)$ & KM4_4 & $\omega_{L}, \omega_{H}, \zeta, \alpha$ & $0.989_{-0.004}^{+0.010}$ & $0.985_{-0.002}^{+0.002}$ & $0.925_{-0.010}^{+0.011}$ & $0.878_{-0.022}^{+0.019}$ \\
\hline $\operatorname{Mix}(M=8)$ & KM8_4 & $\omega_{L}, \omega_{H}, \zeta, \alpha$ & $0.989_{-0.004}^{+0.010}$ & $0.985_{-0.002}^{+0.002}$ & $0.927_{-0.010}^{+0.010}$ & $0.882_{-0.023}^{+0.017}$ \\
\hline $\operatorname{Mix}(M=16)$ & KM16_4 & $\omega_{L}, \omega_{H}, \zeta, \alpha$ & $0.989_{-0.004}^{+0.009}$ & $0.985_{-0.002}^{+0.002}$ & $0.928_{-0.009}^{+0.011}$ & $0.882_{-0.020}^{+0.018}$ \\
\hline $\operatorname{Mix}(M=32)$ & KM32_4 & $\omega_{L}, \omega_{H}, \zeta, \alpha$ & $0.989_{-0.004}^{+0.008}$ & $0.986_{-0.002}^{+0.002}$ & $0.929_{-0.010}^{+0.011}$ & $0.883_{-0.021}^{+0.017}$ \\
\hline $\operatorname{Mix}(M=64)$ & KM64_4 & $\omega_{L}, \omega_{H}, \zeta, \alpha$ & $0.990_{-0.004}^{+0.010}$ & $0.986_{-0.002}^{+0.002}$ & $0.928_{-0.010}^{+0.011}$ & $0.882_{-0.022}^{+0.017}$ \\
\hline $\operatorname{Mix}(M=128)$ & KM128_4 & $\omega_{L}, \omega_{H}, \zeta, \alpha$ & $0.990_{-0.004}^{+0.008}$ & $0.986_{-0.002}^{+0.002}$ & $0.929_{-0.010}^{+0.009}$ & $0.884_{-0.021}^{+0.016}$ \\
\hline $\operatorname{Mix}(M=2)$ & KM2_3cr & $\omega_{c}, P_{c}, \alpha$ & $0.959_{-0.004}^{+0.004}$ & $0.917_{-0.010}^{+0.008}$ & $0.938_{-0.004}^{+0.005}$ & $0.919_{-0.006}^{+0.007}$ \\
\hline $\operatorname{Mix}(M=64)$ & KM64_3cr & $\omega_{c}, P_{c}, \alpha$ & $0.965_{-0.004}^{+0.004}$ & $0.928_{-0.009}^{+0.007}$ & $0.934_{-0.004}^{+0.004}$ & $0.908_{-0.006}^{+0.005}$ \\
\hline
\end{tabular}

In Figure 13, we show the box-plots for the comparison of identification rates among these models, and list their identification rate values in Table 2. It is clear that our model has the highest rate in completeness, especially $99.3 \%$ in the cases of 'Inf4', 'Inf3oz' and 'Inf2zl', and we've confirmed those of Kelly's model are also high compared to other models. It is very plausible that ours and Kelly's models are more flexible model for describing QSO/AGN variability. The significance of the difference in completeness from those of other models such as Butler \& Bloom (2011) and $\mathrm{Zu}$ et al. (2011) models is verified by the pairwise multiple comparison test(Demsar 2006) ${ }^{7}$. In terms of recall, single OU model by $\mathrm{Zu}$ et al. (2011) shows the highest score, but the score of our model is higher than those of other models.

It should be noted that the improvement of identification rates of QSOs, especially in relation to completeness, by our model compared to others comes from the discrimination ability on the objects at the edge of the Group1.

In Figure 14, we show the three-dimensional distribution of our model parameters for spectroscopically confirmed QSOs with the success rates by Butler \& Bloom (2011)'s model and JAVELIN. By comparing the success rates distribution by our model, shown in the middle of Figure 11, it is clear that most objects in the group(Group1) are classified successfully as 'QSO' in our model, although some misclassifications can be seen in the results by other models. It is therefore reasonable to explain this improvement is caused by the introduction of the mixed OU processes in our model.

We should, however, be cautious regarding the bad success rate on 'Inf2zh', which is classified based on $\omega_{H}$ and $\zeta$, instead of very high performance on 'Inf2zl'. It implies that QSOs with small damping time scale are indistinguishable from stars on the parameter plane defined by $\omega_{H}$ and $\zeta$. It should be noted that the larger number of mixtures of OU processes provide better success rates in completeness, although saturated around $M=8$.

In Figure 15, we show the distribution of success rates of QSOs and stars in our 1,000 trials of classification using linear SVM. We compare the performance of the 'Inf4' model to others ('Inf3cr', 'JAVELIN', and 'Butler') in this

${ }^{7}$ http://finzi.psych.upenn.edu/library/PMCMR/html/PMCMR-package.html 

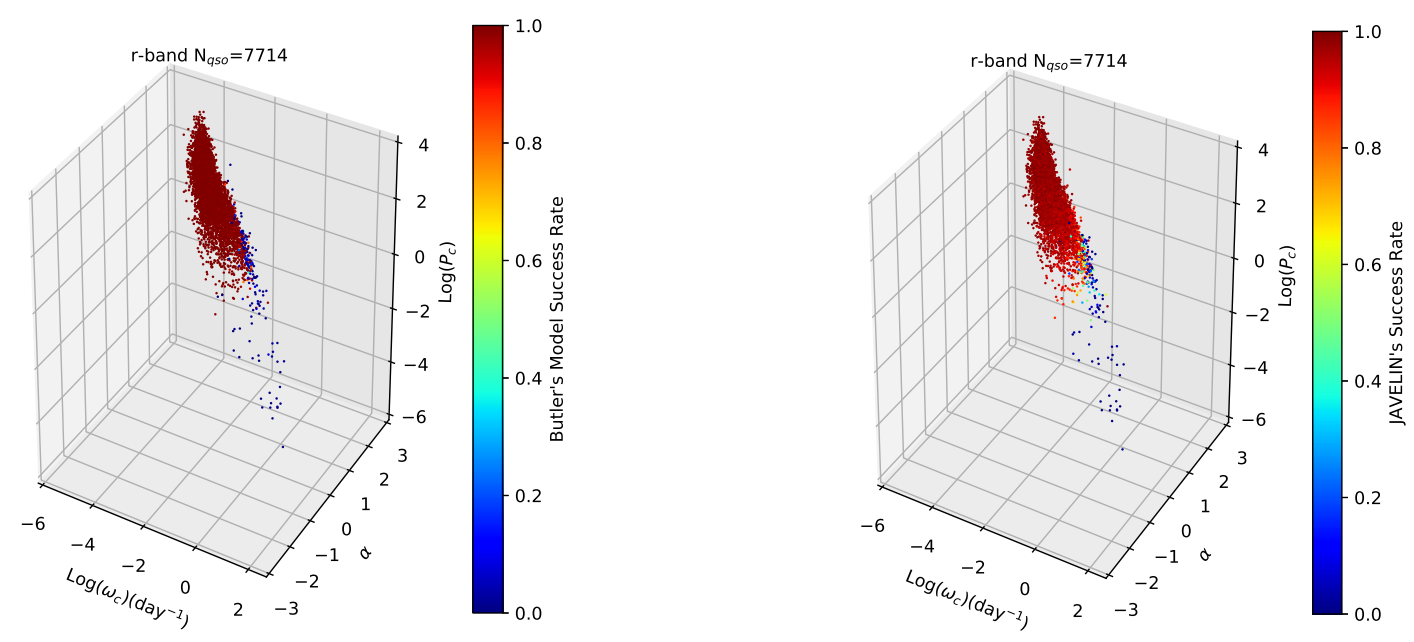

Figure 14. The same one with middle panel of Figure 8, but with the success rates with Butler \& Bloom (2011)'s model(left) and JAVELIN(right).

figure. The identification rates are computed for the whole 'good sample', on 9,855 sources. In this figure we can see the clear advantage the 'Inf4' model has over the 'Butler' model in QSOs and stars selection, and over 'JAVELIN' in QSO selection in the high identification-rate region. On the other hand, we cannot see any significant difference from 'Inf3cr'.
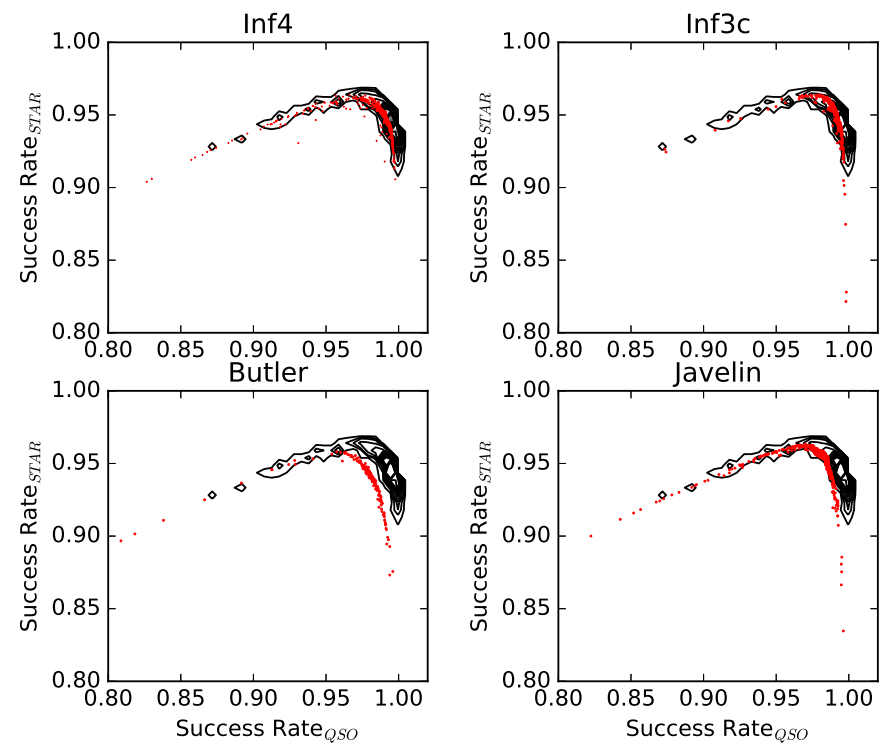

Figure 15. Comparison of identification rates of QSOs and stars for 1000 sets of coefficients in our SVM analysis. Contours in each panel show the distribution for the 'Inf4' model, and the red points represent the results of 1000 trials for each model. They are for Inf4, Inf3cr, Butler's model, and Javelin(DRW model), respectively, in the order of upper left, upper right, lower left, and lower right. 
As we use linear SVM for our classification, the hyperplane separating the parameter zones for QSOs and stars can be expressed as follows;

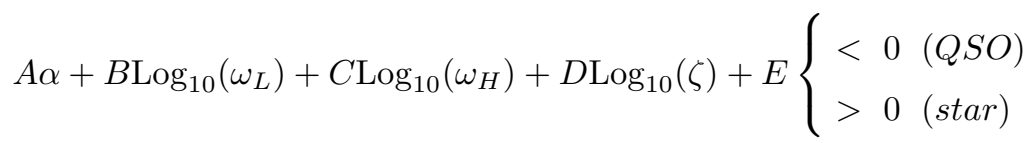

As the number fraction of QSOs to stars depends on sky region and/or depth of the imaging data/catalog, it is convenient to use different hyperplanes for selecting QSOs from the variable object samples, as the 'total' success rates, defined as the weighted mean of success rates for QSO and star selections. The information may be one of the important issues to decide the strategy for QSO selection using the given data set. For example, we should use conservative criteria to eliminate contamination by stars, if the purity of QSO selection is the highest priority on the selection.

Table 3. Coefficients of SVM hyperplane to separate QSOs and stars with best weighted identification rate for given number fractions of QSOs and stars.

\begin{tabular}{rcccccccc}
\hline \hline $\mathrm{N}_{Q S O} / \mathrm{N}_{\text {star }}$ & $\mathrm{A}$ & $\mathrm{B}$ & $\mathrm{C}$ & $\mathrm{D}$ & $\mathrm{E}$ & $\mathrm{R}_{\text {total }}{ }^{a}$ & $\mathrm{R}_{Q S O}{ }^{b}$ & $\mathrm{R}_{\text {star }}{ }^{b}$ \\
& & & & & & & & \\
\hline 0.1 & -0.728 & 0.063 & 0.227 & -0.069 & 1.297 & 0.971 & 0.870 & 0.981 \\
0.5 & -0.664 & 0.139 & 0.211 & -0.019 & 1.209 & 0.965 & 0.957 & 0.970 \\
1.0 & -0.775 & 0.146 & 0.201 & 0.151 & 1.560 & 0.964 & 0.966 & 0.961 \\
1.2 & -0.695 & 0.119 & 0.255 & -0.073 & 0.945 & 0.965 & 0.977 & 0.950 \\
1.5 & -0.695 & 0.119 & 0.255 & -0.073 & 0.945 & 0.966 & 0.977 & 0.950 \\
2.0 & -0.774 & 0.189 & 0.222 & 0.172 & 1.551 & 0.968 & 0.983 & 0.939 \\
2.5 & -0.768 & 0.210 & 0.250 & 0.111 & 1.467 & 0.971 & 0.987 & 0.931 \\
3.0 & -0.086 & 0.425 & 0.395 & -0.410 & 0.949 & 0.972 & 0.988 & 0.924 \\
3.0 & -0.768 & 0.210 & 0.250 & 0.111 & 1.467 & 0.973 & 0.987 & 0.931 \\
5.0 & -0.575 & 0.285 & 0.253 & -0.101 & 1.159 & 0.978 & 0.991 & 0.913 \\
\hline
\end{tabular}

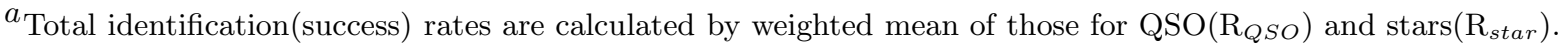

${ }^{b} \mathrm{QSO}\left(\mathrm{R}_{Q S O}\right)$ and $\operatorname{stars}\left(\mathrm{R}_{\text {star }}\right)$ are values of completeness.

We show some sets of hyperplane coefficients for separating QSOs and stars in four parameters space with maximum total success rates (weighted mean of QSOs and stars success rates) in Table 3 with an assumed number fraction of QSOs to stars in the sample. Note that in our photometric sample, the fraction varies from about 0.1 to 2.2. For example, in case we apply the coefficients for $\mathrm{N}_{Q S O} / \mathrm{N}_{\text {star }}=1.5$, which is the rough average of the data set, we select 9,077 QSO candidates out of 23,677 variable sources, which have more than 40 measurements with errors less than 0.05 magnitudes, based only on photometric data. In this case it will provide additional $\sim 1,300$ QSO candidates to the current spectroscopically confirmed QSOs in the data set.

\subsection{Failure rates and their meanings}

In our analysis, we identified 83 spectroscopically confirmed QSOs with the 'false' classification by their optical variability. The 'false' means the success rate is lower than $50 \%$ in our analysis. It is about one percent of the spectroscopically confirmed QSOs and it is interesting to know the reason for this failure in the classification. We visually inspect all the spectra of 83 QSOs, and confirm that 79 objects can be reasonably classified as QSO/AGN. The remaining four objects are three stars and one object with failure in taking the spectrum(fiber allocation might have failed). It should be noted that the misclassification occurs on the common objects by the analysis based on 
other models, such as Butler's model, single-OU and Kelly's mixture model, and it is not a characteristic feature of our model. It means that their variability can not be distinguished from those of stars.

There are several possibilities for explaining the failure of classification. The first is the effect caused by the irregularly taken time series data, that hides or dulls the emergence of the QSO/AGN-like variability features in their light curves. It may be coming from the limitation of MCMC procedure for producing the light curves for our stochastic model analysis. It is however difficult to consider this a plausible reason, as the most of objects are successfully classified as 'QSO' in spite of being under the conditions of very similar cadence, as described in 3.2.

The second possibility is that this discrepancy comes from the time difference between photometric and spectroscopic observations. As we are based on the SDSS DR12 data on the spectroscopic data, although the photometric data is coming from DR7, there is a possibility of a change of physical condition in these objects. We investigate the MJDs of the data taking on photometry and spectroscopy, and confirm that 40 and 43 spectral data are taken in and out of the duration on the photometric data. Therefore, it is difficult to consider the time difference of photometry and spectroscopy as the main reason for the discrepancy.

The third and most plausible is that the objects are rare type QSOs/AGNs whose variability features differ from those of most QSOs. There may be the signatures of (quasi-) periodic features in their light curves and/or cannot be well described by MCMC procedure as mentioned above.

As shown in the left panel of Figure 16(a), objects classified as not 'QSO' (categorized in Group2 as described in 4.2) have single-bending PSDs with very large $\omega$ at the bending points, which is very similar to those for stars in our analysis. We also show the light curve of the same object in Figure 16(b), and it is clear that the variation in short time scale is so large and we cannot see the damping of brightness, which is seen in typical QSOs, as shown in Figure 2. This implies the rarity (they contribute only one percent of spectroscopically confirmed QSOs) of this QSO in terms of optical variability, and there might be a different physical process controlling the variation of brightness, at least in a short time scale.

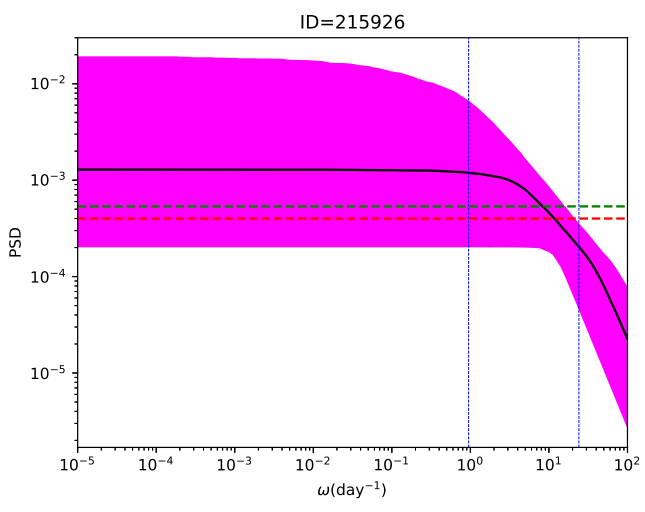

(a)

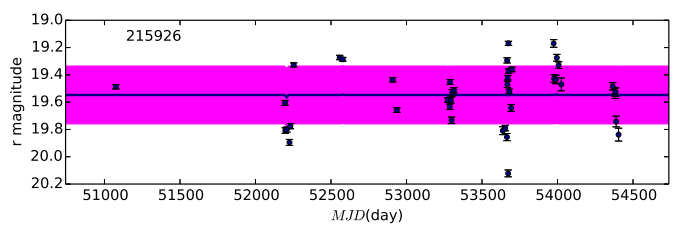

(b)

Figure 16. (a) A PSD of a spectroscopically confirmed QSO categorized in Group2, which can not be distinguished from those of stars. (b) Light curve of the same object in $r$ band magnitudes.

We cannot see any significant difference in the spectra of the QSOs with 'false' classification, compared to the typical variable QSOs, and it is plausible to consider the discrepancies as coming from the physical processes which are effective to optical variability although not to optical spectral features.

As the fractions of QSOs and stars spectroscopically misidentified are so small (4/83 for QSOs and 16/199 for stars, respectively), it is clear that the results of identification rates discussed in previous sections are not affected seriously.

\subsection{Features of our model and Interpretation of QSOs' PSD parameters}

We compare the goodness of the fit of our model to single OU process(DRW) model by using AIC and BIC(Bayesian information criterion)(Schwarz 1978).

$\mathrm{AIC}$ is derived by the following equation, 


$$
A I C=-2 \ln (L)+2 k,
$$

where $L$ is the maximum value of likelihood function and $k$ is the number of estimated parameters in the model. In the case of our model, $k$ is 4 , and $k$ is 2 for DRW model. BIC is derived by the Equation 25 as described in 4.1 .

In Figure 17, we show the comparison of AICs and BICs for QSOs in the 'good sample'. It is clear that our model works better for QSOs with large differences between $\omega_{L}$ and $\omega_{H}$, which correspond to variability different from DRW, since the smaller AIC/BIC values mean better fitness.

The trends seen about AICs are the same for BICs, that our model works better for QSOs with larger discrepancies between $\omega_{L}$ and $\omega_{H}$, for example $\log \frac{\omega_{L}}{\omega_{H}} \leq-3$.

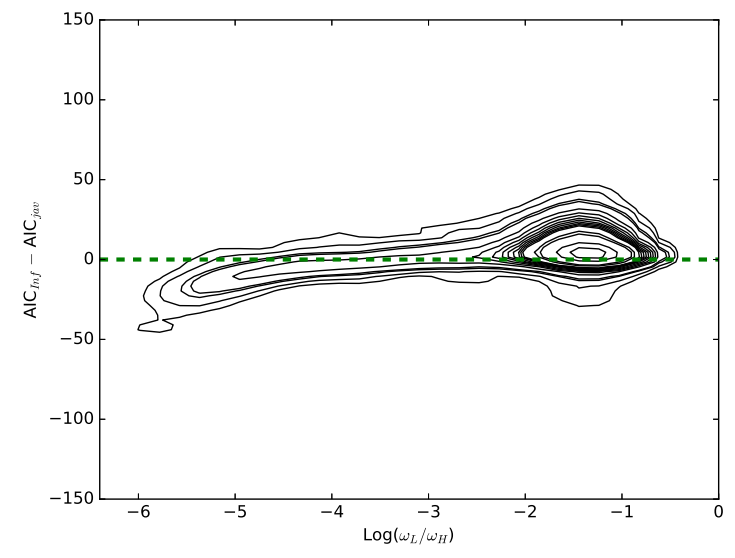

(a)

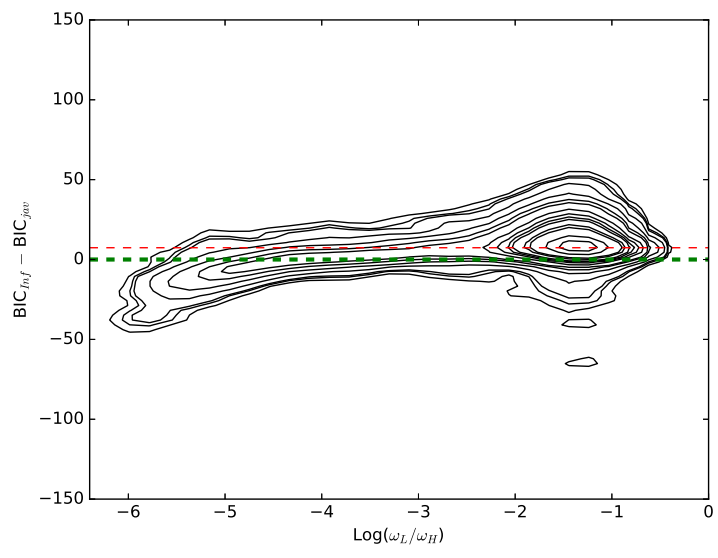

(b)

Figure 17. (a)The dependencies of the differences of AIC values between those for our model and DRW model, to the ratio of $\omega_{L}$ and $\omega_{H}$ in our model. The horizontal green dashed line shows the border for equal AIC values, which means the two models have the same rank. (b)The same figure for BIC values. The horizontal green line means the same as 17(a), and red dashed line show the case when the likelihoods are the same in the case number of measuremets is 40 .

It is therefore reasonable to think our model provides better fit than those based on DRW model for QSOs with larger differences between $\omega_{L}$ and $\omega_{H}$. It should be noted that many QSOs, whose variability can be well described by DRW model, show worse fitting results in our model. However, this causes no significant effect for selection of QSOs/AGNs from variable stars. The differences are only coming from the number of model parameters, and the likelihood values are equivalent about both models.

We also note here that the AIC in its original form is strictly only valid asymptotically, and the correction to AIC for finite sample sizes suggested by Sugiura (1978) and Hurvich \& Tsai (1989), commonly denoted as c-AIC, may be used for the discussions. We also check the c-AIC and confirm that there is no difference from the result by using AIC.

The PSDs for the QSOs/AGNs with smaller AIC/BIC in our model show the power law like features, which have flatter slopes than $\beta=-2$, when we express the PSD $\propto \omega^{\beta}$. In most cases they have slopes $\beta \sim-1.8$ or so. They have very small $\omega_{L}\left(\log \omega_{L} \sim-4\right)$ and large $\omega_{H}\left(\log \omega_{L} \sim 1-2\right)$ as shown 'PowerLaw' type variability in Figure $5(\mathrm{~b})$. In our model such a variability can be expressed well than DRW model by selecting large difference in $\omega_{L}$ and $\omega_{H}$.

On the other hand, most QSO have small differences between $\omega_{L}$ and $\omega_{H}$, typically $\log \omega_{L} / \omega_{H} \sim-1$. In our model there is no constraint about the values of $\omega_{L}$ and $\omega_{H}$, except for $\omega_{L} \leq \omega_{H}$. One of the reason for this small diffference is our method for estimating model parameters. As we use median values of posteriors $\left(\omega_{L}\right.$ and $\left.\omega_{H}\right)$, which have distributions in a certain level, and we use the constraint of $\omega_{L} \leq \omega_{H}$ in our calculation, it is reasonable to get some difference between $\omega_{L}$ and $\omega_{H}$. Since the typical errors of parameter inferences are 0.4 dex in the case, it is plausible to consider this is the main reason for the small diffferences. This guess can be supported by the fact that the slope of the distribution of these objects in Figure 5(b) is well aligned to that of 'ideal' DRW model's one as shown by green 
dotted lines. Since the data we used for the analysis is not dense in time, it is very difficult to discuss the hypothesis of their intrinsic origin.

\subsection{Issues for analyzing more massive data with our model}

In our model the calculation time is the order of $O\left(N^{2}\right)$, when $N$ is the number of observed data points. Compared to Kelly's OU mixture model, which needs time with the order of $O\left(M N^{2}\right)(M$ is the numbers of mixed OU processes), our model has an advantage in calculation time. However, it is still very massive for the data with numerous measurements, or with many observed objects. If we limit our analysis to those based on the stationary process, we may implement the methods based on phase-space description or Kalman filter for reducing the calculation time. Using the well-known time series analysis methods like ARMA (Auto-Regression Moving Average) model, as suggested by Kelly et al. (2014); Simm et al. (2016); Kasliwal et al. (2017), is among useful candidates for the massive data time series analysis in near future. It is, however, plausible to consider that some non-stationary processes also affect the optical variability of QSO/AGN. For analyzing time series data of QSO/AGN with a more complicated model, we should continue to strive for the effective algorithms using powerful computational equipment like many CPU cores and supportive database systems.

\section{CONCLUSIONS}

We develop the infinite mixture model of the OU process for describing the optical variability of QSOs/AGNs, which enables us to get the results within an appropriate time. The main reason for this faster calculation is our analytical deriving of the covariance function. It is based on the consideration for treating the variability as a stochastic process, and it enables us to get the parameters on their PSDs for their brightness variations.

We apply our model to 67,507 objects extracted from SDSS Stripe82 photometric data with sufficient multiple measurements, and succeed in showing very high precision in selecting QSOs among variable objects based only on their variability, by investigating $\sim 10,000$ spectroscopically confirmed objects. This can be a good first step for enabling the classification and investigating the physical processes among various types of QSO/AGN using their variability.

We find out that QSO/AGN variability can be well described in a certain regular manner in our model parameter space on their PSDs on QSO/AGN variability, with very few outliers. In general, the smaller the amplitude of variation, the shorter the damping time scale, which is consistent with the previous studies. The majority $(95 \%)$ of spectroscopically confirmed QSOs may be well described by DRW model. However, four percent of QSOs belong to the group with large differences between $\omega_{L}$ and $\omega_{H}$. The remaining one percent of QSOs are indistinguishable from stars in our model.

Our model is more flexible than other previously suggested models based on a single OU process (damped random walk), as we can describe their PSDs of variability with more accuracy. We eliminate the arbitrariness in the number of mixed OU processes from the original model, and our implementation enables calculations to finish within tolerable time. We show that this type of analysis is feasible in an era with more massive data, though more calculation speed is desirable.

Based on our model, we try the separation of QSOs and stars based only on the variability, and succeed in identifying QSOs with $99.3 \%$ completeness and $99.0 \%$ purity. These numbers show our model can be used as the most effective method for selecting QSOs from a variable object catalog, and is superior to other models in terms of completeness. Other evaluations of the identification capabilities, such as accuracy and recall, show mostly the same levels as those for other competitive models, which are widely used and discussed for identifying QSOs based only on the variability. The main reason supporting our model's superiority in terms of QSO selection comes from our introduction of the mixture of OU processes, which provides more flexibility on the description of PSDs and enables us to separate raretype QSOs/AGNs, which can not be well dexscribed by simple DRW model, from variable stars in the parameter space.

We thank all the people who supported the research in many phase, especially to Prof. Yoshiyasu Tamura, Prof. Hidetoshi Konno, Prof. Shinichiro Koyama, Prof. Isao Shoji, Dr. Masaya Saito and Dr. Yuya Ariyoshi for their fruitful discussions in the preparation of the paper. 
This research was supported by the internship program of "Data Scientist Training Network" 8 , a Commissioned Projects by The Ministry of Education, Culture, Sports, Science and Technology of Japan, and was also supported by JSPS KAKENHI Grant Number JP22012007.

Funding for the SDSS and SDSS-II has been provided by the Alfred P. Sloan Foundation, the Participating Institutions, the National Science Foundation, the U.S. Department of Energy, the National Aeronautics and Space Administration, the Japanese Monbukagakusho, the Max Planck Society, and the Higher Education Funding Council for England. The SDSS Web Site is http://www.sdss.org/.

The SDSS is managed by the Astrophysical Research Consortium for the Participating Institutions. The Participating Institutions are the American Museum of Natural History, Astrophysical Institute Potsdam, University of Basel, University of Cambridge, Case Western Reserve University, University of Chicago, Drexel University, Fermilab, the Institute for Advanced Study, the Japan Participation Group, Johns Hopkins University, the Joint Institute for Nuclear Astrophysics, the Kavli Institute for Particle Astrophysics and Cosmology, the Korean Scientist Group, the Chinese Academy of Sciences (LAMOST), Los Alamos National Laboratory, the Max-Planck-Institute for Astronomy (MPIA), the Max-Planck-Institute for Astrophysics (MPA), New Mexico State University, Ohio State University, University of Pittsburgh, University of Portsmouth, Princeton University, the United States Naval Observatory, and the University of Washington.

Funding for SDSS-III has been provided by the Alfred P. Sloan Foundation, the Participating Institutions, the National Science Foundation, and the U.S. Department of Energy Office of Science. The SDSS-III web site is http://www.sdss3.org/.

SDSS-III is managed by the Astrophysical Research Consortium for the Participating Institutions of the SDSS-III Collaboration including the University of Arizona, the Brazilian Participation Group, Brookhaven National Laboratory, Carnegie Mellon University, University of Florida, the French Participation Group, the German Participation Group, Harvard University, the Instituto de Astrofisica de Canarias, the Michigan State/Notre Dame/JINA Participation Group, Johns Hopkins University, Lawrence Berkeley National Laboratory, Max Planck Institute for Astrophysics, Max Planck Institute for Extraterrestrial Physics, New Mexico State University, New York University, Ohio State University, Pennsylvania State University, University of Portsmouth, Princeton University, the Spanish Participation Group, University of Tokyo, University of Utah, Vanderbilt University, University of Virginia, University of Washington, and Yale University.

8 http://datascientist.ism.ac.jp/ 


\section{REFERENCES}

Akaike, H. 1973, Proceedings of the Second International Symposium on Information Theory, ed. B. Petrov \& F. Csaki (Budapest: Akademiai Kiado), 267

Alam, S., Albareti, F. D., \& Allende P. C. et al. 2015, ApJS, 219, 12

Andrae, R., Kim, D.-W., \& Bailer-Jones, C. A. L. 2013, A\&A, 554, 137

Abazajian, K. N., Adelman-McCarthy, J. K., Agueros, M. A. et al. 2009, ApJS, 182, 543

Bhattacharyya, A. 1943, Bulletin of the Calcutta Mathematical Society. 35: 99109

Brockwell, P. J. \& Davis, R. A. 2002, Introduction to Time Series and Forecasting (2nd ed.; New York: Springer)

Butler, N., \& Bloom, J. 2011, AJ, 141, 93

Caplar, N., Lilly, S. \& Trakhtenbrot, B. 2017, ApJ, 834, 111

Carini, M. T. \& Ryle, W. T. 2012, ApJ, 749, 70

Cartier, R., Lira, P., Coppi, P., et al. 2015, ApJ, 810, 164

Chen, X-Y., \& Wang, J-X. 2015, ApJ, 805, 80

Choi Y., Gibson R. R., Becker A. C., et al. 2014, ApJ, 782, 37

De Cicco, D., Paolillo, M., Covone, G., et al. 2015, A\&A, 574, A112

Demsar, J 2006, Statistical comparisons of classifiers over multiple data sets, Journal of Machine Learning Research, 7, 1-30

Dexter, J., \& Agol, E. 2011, ApJL, 727, L24

Edelson, R., Vaughan, S., \& Malkan, M. et al. 2014, ApJ, 795,2

Gaskell, C. M., \& Klimek, E. S. 2003, Astron. Astrophys. Trans., 22, 661

Gonzlez-Martn, O. \& Vaughan, S. 2012, A\&A, 544, A80

Graham, M. J., Djorgovski, S. G. \& Drake, A. J. et al. 2014, MNRAS, 439, 703

Greenstein J. L. 1963, Nature, 197, 1041

Gunn, J. E., Carr, M., Rockosi, C.,Sekiguchi, M. et al. 1998 AJ, 116, 3040

Falocco, S., Paolillo, M., Covone, G., et al. 2015, A\&A, 579, A115

Foreman-Mackey, D., Hogg, D.W., Lang, D. \& Goodman, J. 2012, arXiv:1202.3665

Hirose, S., Krolik, J. H., \& Blaes, O. 2009, ApJ, 691, 16

Hughes, P. A., Aller, H. D. \& Aller, M. F. 1992, ApJ, 396, 469

Hurvich, C. M. \& Tsai, C.-L. 1989, Biometrika, 76, 297 Ivezić, Z̃., et al. 2007, AJ, 134, 973

Jiang, Y.-F., Stone, J. M., \& Davis, S. W. 2013, ApJ, 767, 148

Kasliwal V. P., Vogeley M. S. \& Richards G. T. 2015a, MNRAS, 451, 4328
Kasliwal V. P., Vogeley M. S., Richards G. T., Williams J. \& Carini M. T. 2015b, MNRAS, 453, 2075

Kasliwal V. P., Vogeley M. S. \& Richards G. T. 2017, MNRAS, 470, 3027

Kelly, B.C., Bechtold, J. \& Siemiginowska, A. 2009, ApJ, 698,895

Kelly, B.C., Sobolewska, M. \& Siemiginowska, A. 2011, ApJ, 730, 52

Kelly, B.C., Treu, T., Malkan, M. et al. 2013, ApJ, 779, 187

Kelly, B.C. et al. 2014, ApJ, 788, 33

Kim, D.-W., Protopapas, P., Byun, Y.-I., et al. 2011, ApJ, 735,68

Kozłowski, S., Kochanek, C. S., \& Udalski, A. et al. 2010, ApJ, 708, 927

Kozłowski, S., Kochanek, C. S., \& Stern, D. et al. 2010, ApJ, 716, 530

Kozłowski, S., Kochanek, C. S., \& Udalski, A. 2011, ApJS, 194,22

Kozłowski, S., Kochanek, C. S., Jacyszyn, A. M. et al. 2012, ApJ, 746, 27

Kozłowski, S., Onken, C. A., Kochanek, C. S. et al. 2013, ApJ, 775, 92

Kozłowski, S. 2016a, MNRAS, 459, 2787

Kozłowski, S., Kochanek, C. S., Ashby, M. L. N. et al. 2016b, ApJ, 817, 119

Kozłowski, S. 2016c, ApJ, 826, 118

MacLeod, C. L., Ivezić, Z̃., \& Kochanek, C. S. et al. 2010, ApJ, 721, 1014

MacLeod, C. L., Brooks, K., \& Ivezić, Z̃. et al. 2011, ApJ, 728,26

MacLeod, C. L., Ivezić, Z̃., \& Sesar, B., et al. 2012, ApJ, 753,106

Markowitz, A., Edelson, R., \& Vaughan, S. et al. 2003, ApJ, 593, 96

Matthews T. A. \& Sandage A. R. 1963, ApJ, 138, 30

McHardy, I. M., Papadakis, I. E., Uttley, P. et al. 2004, MNRAS, 348, 783

Morganson, E., Burgett, W. S., Chambers, K. C., et al. 2014, ApJ, 784, 92

Mushotzky, R. F., Edelson, R., \& Baumgartner, W. et al. 2011, ApJL, 743, L12

Palanque-Delabrouille, N., Yeche, C., Myers, A. D., et al. 2011, A\&A, 530, A122

Pancoast A., Brewer B. J. \& Treu T. 2014, MNRAS, 445, 3055

Pereyra, N. A., Van den Berk, D. E., Turnshek, D. A., et al. 2006, ApJ, 642, 87

Peters, C. M., Richards, G. T., Myers, A. D., et al. 2015, ApJ, 811, 95 
Press W. H., Rybicki G. B. and Hewitt J. N. 1992, ApJ385, 404

Plotkin, R. M., Anderson, S. F. \& Brandt, W. N. et al. 2010, AJ, 139, 390

Revalski, M., Nowak, D., \& Wiita, P. J. et al. 2014, ApJ, 785,60

Ruan J. J., Anderson S. F., MacLeod C. L. et al.. 2012, ApJ, 760, 51

Rybicki G. B. and Press W. H. 1992, ApJ, 398, 169

Rybicki G. B. and Press W. H. 1995, PhRvL, 74, 1010

Schmidt M. 1963, Nature, 197, 1040

Schmidt, K. B., Marshall, P. J., Rix, H.-W., et al. 2010, ApJ, 714, 1194

Schmidt, K. B., Rix, H.-W., Shields, J. C., et al. 2012, ApJ, 744, 147

Schwarz, G. 1978, Annals of Statistics, 6(2), 461

Sasar, B., et al. 2007, AJ, 134, 2236

Shaya, E. J., Olling, R., \& Mushotzky, R. 2015, AJ, 150, 188

Simm T., Salvato M., Saglia R. et al 2016, A\&A, 585, A129
Smith H. J. \& Hoffleit D. 1963, Nature, 198, 650

Sugiura, N. 1978, "Further analysts of the data by akaike' s information criterion and the finite corrections", Communications in Statistics - Theory and Methods, $7(1)$, pp. $13-26$

Uhlenbeck, G.E. \& Ornstein, L.S. 1930, PhRvA, 36, 823

Uttley, P., McHardy, I. M., \& Papadakis, I. E. 2002, MNRAS, 332, 231

Uttley, P., \& Casella, P. 2014, Space Sci. Rev., 183, 453

Wehrle, A. E., Wiita, P. J. \& Unwin, S. C. et al. 2013, ApJ, 773, 89

Zhu, S. F., \& Xue, Y. Q. 2016, ApJ, 825, 56

Zu, Y., Kochanek, C.S., \& Peterson, B.M. 2011, ApJ, 735, 80

Zu, Y., Kochanek, C.S., Kozłowski, S., \& Udalski, A. 2013, ApJ, 765, 106

Zuo, W., Wu, X.-B., Liu, Y.-Q., \& Jiao, C.-L. 2012, ApJ, 758,104 